\title{
Flow of a viscous nematic fluid around a sphere
}

\author{
Manuel Gómez-González *1 and Juan C. del Álamo, \\ ${ }^{1}$ Mechanical \& Aerospace Engineering Department, University of \\ California San Diego. \\ ${ }^{2}$ Institute for Engineering in Medicine, University of California San \\ Diego.
}

June 18, 2021

\begin{abstract}
We analyze the creeping flow generated by a spherical particle moving through a viscous fluid with nematic directional order, in which momentum diffusivity is anisotropic and which opposes resistance to bending. Specifically, we provide closed-form analytical expressions for the response function, i.e. the equivalent to Stokes's drag formula for nematic fluids. Particular attention is given to the rotationally pseudo-isotropic condition defined by zero resistance to bending, and to the strain pseudo-isotropic condition defined by isotropic momentum diffusivity. We find the former to be consistent with the rheology of biopolymer networks and the latter to be closer to the rheology of nematic liquid crystals. These "pure" anisotropic conditions are used to benchmark existing particle tracking microrheology methods that provide effective directional viscosities by applying Stokes's drag law separately in different directions. We find that the effective viscosity approach is phenomenologically justified in rotationally isotropic fluids, although it leads to significant errors in the estimated viscosity coefficients. On the other hand, the mere concept of directional effective viscosities is found to be misleading in fluids that oppose an appreciable resistance to bending. Finally, we observe that anisotropic momentum diffusivity leads to asymmetric streamline patterns displaying enhanced (reduced) streamline deflection in the directions of lower (higher) diffusivity. The bending resistance of the fluid is found to modulate the asymmetry of streamline deflection. In some cases, the combined effects of both anisotropy mechanisms leads to streamline patterns that converge towards the sphere.
\end{abstract}

*manu@ucsd.edu 


\section{Introduction}

Nematic fluids exhibit molecular or supramolecular alignment along a director vector field, leading to anisotropic rheology. These fluids appear often in industrial applications and in biology. For instance, nematic liquid crystals are a key component in a vast variety of displays for their high resolution and energy efficiency. The interaction between flow, topological defects and nematic orientation in liquid crystals are exploited in particle self-assembly (Poulin et al., 1997, Loudet et al., 2000) and sensing applications (Brake et al., 2003). Anisotropic rheology is also observed in reconstituted gels when the polymer phase is aligned (Hasnain and Donald, 2006 He et al. 2008) and, perhaps more importantly, the cytoplasm of animal cells is far from being isotropic. The filaments that make up the cytoskeleton can experience spatial order and alignment both at the level of the mesh size $(\sim 10 \mathrm{~nm})$ and of the whole cell $(\sim 10 \mu \mathrm{m})$, leading to short- and long-range directionality (Luby-Phelps, 2000). Intracellular rheology has been proposed to modulate important cell functions such as mechanotransduction, (Chien, 2007; Wang et al., 1993, Chen et al., 2004), cell migration (Kole et al. 2005 Pollard and Borisy, 2003 Yanai et al., 2004) and intracellular organelle transport (Lammerding and Lee, 2005; Lee et al., 2005; Minin et al., 2006). Recent studies suggest that intracellular anisotropy may play an important role in controlling the directionality of these cellular processes (Scherp and Hasenstein, 2007; Rogers et al. 2008; del Álamo et al., 2008). In short, anisotropy is purpose.

Particle Tracking Microrheology (PTM) determines the viscoelastic shear modulus of a medium from the measured motion of embedded microparticles (Mason and Weitz, 1995, Mason et al., 1997; Mason, 2000). This technique is particularly suitable for probing minute quantities of biological materials, including live cells (Tseng et al., 2002, Crocker and Hoffman, 2007, del Álamo et al. 2008). In active PTM, the motion of the probing particle is forced with magnetic or laser tweezers, whereas in passive PTM this motion is caused by the thermal excitation of the medium. These two approaches estimate the complex response function $\tilde{\zeta}(s)$ that relates the drag force experienced by the probing particle and the particle velocity as $\tilde{\mathbf{f}}(s)=\tilde{\zeta}(s) \tilde{\mathbf{v}}(s)$, where $\tilde{r}$ indicates Laplace transform and $s$ is the Laplace frequency. A crucial step in both active and passive PTM is to connect the measured response function to the underlying rheological properties of the medium, which is usually accomplished by assuming that the particle experiences a generalized Stokes's resistance. For a sphere of radius $a$ moving in a single-phase no-slip isotropic continuum, the response function is modeled as $\tilde{\zeta}(s)=6 \pi a \tilde{\eta}(s)$ (Mason and Weitz, 1995, Squires and Mason, 2010), where $\tilde{\eta}(s)$ is the frequency dependent viscosity of the medium.

The application of the generalized Stokes's law in complex biomaterials (e.g. gels) is complicated by their multiphase nature as well as by interactions between the material and the probing particle. For instance, the Stokes's flow assumption can break down in live cells when tracking endogenous particles that are anchored to the cytoskeleton (Lin et al., 1990). Furthermore, the mobility of injected microspheres has been reported to vary dramatically depending 
on their surface charge (Tseng et al., 2002). Chemical interactions, polymer depletion near the microsphere and network compressibility can also introduce substantial deviations from Stokes's flow (Squires, 2008, McGrath et al., 2000. Valentine et al., 2004, Gittes et al., 1997).

Theoretical studies have analyzed the effect of both network compressibility and slip between the probing particle and the material in two-fluid gels composed by a polymer network viscously coupled to a solvent (Levine and Lubensky, 2000, Fu et al., 2008). These studies have provided frequency-dependent corrections to the generalized Stokes's law that can be used to better interpret PTM experiments. The introduction of two-point PTM (Crocker et al. 2000. Levine and Lubensky, 2001) has allowed investigators to account for most nonStokesian effects in the vicinity of the probing particle by cross-correlating the motion between pairs of distant probing particles.

Despite these advances, little is known about the effect of anisotropy in the response function of a microrheology probe. This lack of knowledge affects both the active and passive modalities of PTM, as well as both single-particle and twoparticle microrheology. Previous studies have provided directional diffusivity coefficients (effective viscosities) of microrheological probes in nematic fluids, $\eta_{\|}^{\text {eff }}$ and $\eta_{\perp}^{\text {eff }}$, where $\|$ and $\perp$ denote, respectively, the directions parallel and perpendicular to the nematic director. Although these measurements provide useful information about anisotropic diffusion processes, it is not clear how to relate $\eta_{\| / \perp}^{\text {eff }}$ with the directional viscosities of anisotropic fluids because the Stokes's formula is not applicable in this case.

In this paper, we address the limitations of PTM in anisotropic fluids by calculating an analytical expression for the response function of a microsphere moving through an orthotropic two-fluid gel. In $\$ 2$, we formulate the mathematical problem under the assumptions that the director remains constant and is not altered by the motion of the sphere (see figure 1) and that there is strong viscous coupling between the network phase and the liquid phase. The anisotropy of the medium is modeled through the Leslie-Ericksen constitutive equations (Ericksen, 1960, Leslie, 1966). Interestingly, the resulting flow equations are also valid for the case of a nematic fluid with high bending elastance and free of defects, thereby conferring a broader reach to the results of this theoretical study.

The Green's function of the problem is obtained in $\$ 3$ and is used in $\$ 4$ to determine an explicit analytical expression for the anisotropic response function that depends on up to four viscosity coefficients. This solution is compared with numerical solutions that have previously been obtained for particular combinations of the viscosity coefficients (Heuer et al., 1992), obtaining excellent agreement. We find that the anisotropy of the Leslie-Ericksen constitutive relation affects the response function through two independent mechanisms. One is the anisotropy in the stress-strain relationship and the other is the bending of the fluid with respect to the nematic director. These mechanisms are studied in isolation by considering rotationally pseudo-isotropic fluids in which the bending stresses are zero, and strain pseudo-isotropic fluids in which the stress-strain relation is isotropic. 


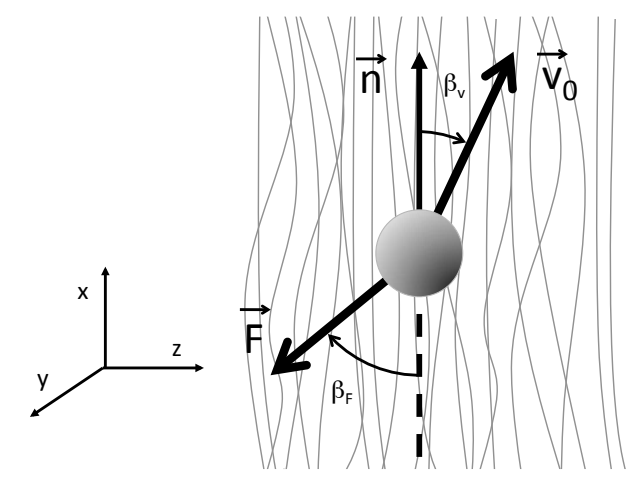

Figure 1: We consider an orthotropic gel with constant and homogeneous nematic. The motion of the sphere is assumed to not alter the nematic.

Analysis of our results and experimental data from nematic biopolymer networks in $\$ 5$ reveals that the application of Stokes's drag law can lead to errors of orders of magnitude in PTM measurements even for moderate levels of anisotropy. Section 6 illustrates the effect of anisotropy on the flow velocity field showing that, in some cases, it leads to streamline patterns that challenge our intuition about creeping flow.

\section{Problem Formulation}

This section introduces the equations to be solved in subsequent sections. We start by considering a two-fluid gel formed by a dilute polymer network that is permeated by a liquid. We illustrate the possible sources of anisotropy in this model before performing a number of simplifying assumptions that hold under the conditions of a live-cell PTM experiment. The simplified equations are still of general scope, as they also describe the flow in other anisotropic fluids such as nematic liquid crystals. We conclude the section by providing a physical interpretation of the different viscosity terms appearing in the flow equations.

As mentioned above, we consider a two-fluid gel composed of a polymer network viscously coupled to a solvent similar to previous PTM studies (Levine and Lubensky, 2000, 2001, Fu et al., 2008). The viscoelastic network is characterized by its displacement field $\vec{u}$, and the viscous solvent by its velocity field $\vec{v}$. We assume that the network is dilute as the polymer network fraction $\phi$ in the eukaryotic cytoskeleton is very small ( $\phi \sim 0.01-0.02$, see Su et al., 2007). For a dilute network, the two-fluid equations take the form

$$
\begin{aligned}
\rho \ddot{\vec{u}}-\nabla \cdot \overline{\bar{\tau}}_{N} & =-\overline{\bar{\Gamma}} \cdot(\dot{\vec{u}}-\vec{v})+\vec{f}_{u}, \\
\rho_{F} \dot{\vec{v}}-\eta \nabla^{2} \vec{v}+\nabla p & =\overline{\bar{\Gamma}} \cdot(\dot{\vec{u}}-\vec{v})+\vec{f}_{v}, \\
\nabla \cdot \vec{v} & =0
\end{aligned}
$$


where $\overline{\bar{\tau}}_{N}$ is the Cauchy stress tensor in the network, $\overline{\bar{\Gamma}}$ is a tensor that represents the viscous coupling between the network and the solvent and $\vec{f}_{u}$ and $\vec{f}_{v}$ model the stresses caused by the sphere in the gel.

The directional order in the network may cause anisotropic dynamics not only through constitutive equations of the polymer but also through the viscous coupling between the polymer and the solvent, even if the solvent is isotropic. For instance, if we idealise the polymer phase in a nematic network as a square array of circular cylinders, then the coupling tensor is defined by the two coefficients

$$
\Gamma_{\|}=\frac{\pi \eta}{\xi^{2} \log (A / \phi)} \quad \text { and } \quad \Gamma_{\perp}=\frac{2 \pi \eta}{\xi^{2} \log (A / \phi)},
$$

where $\xi$ is the distance between neighbouring filaments and $\mathrm{A}$ is a non-dimensional shape factor that takes the value $A \approx 0.23$ under dilute conditions $(\phi<0.02$, see Wang, 2003). Note that, in this case, the viscous coupling is weaker in the nematic direction than in the perpendicular directions by a factor of two.

An order-of-magnitude analysis of equation (2) after eliminating the pressure indicates that viscous coupling is dominant for $(k \xi)^{2} \ll 1$ and $s \xi^{2} \rho_{F} / \eta \ll$ 1 , where $k$ and $s$ are respectively a characteristic wavenumber and frequency. These conditions are often met in live cell cytoplasmic microrheology, where the network (cytoskeleton) mesh size is $\xi \sim 0.02 \mu \mathrm{m}$, and the density and viscosity of the cytosol (the solvent) are approximately equal to those of water (LubyPhelps et al., 1986: Luby-Phelps, 2000). Using the inverse radius of the probing particle $1 / a$ as an upper bound to $k$, the strong coupling limit is found for $a \gg \xi \sim 0.02 \mu \mathrm{m}$ and $s \ll 10^{4} \mathrm{~s}^{-1}$. In the forthcoming analysis, we assume that these conditions hold so that $\dot{\vec{u}}=\vec{v}$, and equations (1) and (2) combine into

$$
\rho \dot{\vec{v}}-\nabla \cdot \overline{\bar{\tau}}=\vec{f},
$$

where $\overline{\bar{\tau}}$ is prescribed, as a function of $\vec{v}$, by the constitutive relations for the strongly coupled two-fluid gel. Interestingly, this simplification makes the flow equations applicable to single-phase fluids such as nematic liquid crystals as well. Note that, due to the strong coupling and the incompressibility of the solvent (3), the network motion in this model is also divergence-free, even if the network is compressible.

Equation 4 is completed with the Leslie-Ericksen constitutive relation, which is generally used for anisotropic fluids with nematic order (Ericksen, 1960, Leslie, 1966: De Gennes and Prost, 1993),

$$
\begin{aligned}
\tau_{i j}= & -p \delta_{i j}+\alpha_{1} n_{k} n_{p} \varepsilon_{k p} n_{i} n_{j}+\alpha_{2} n_{i} N_{j} \\
& +\alpha_{3} n_{j} N_{i}+\alpha_{4} \varepsilon_{i j}+\alpha_{5} n_{i} n_{k} \varepsilon_{k j}+\alpha_{6} n_{j} n_{k} \varepsilon_{k i} .
\end{aligned}
$$

This constitutive relation depends on the six Leslie viscosity coefficients, $\alpha_{i}$. It relates the stress tensor $\tau_{i j}$ with the strain rate tensor $\epsilon_{i j}=\left(v_{i, j}+v_{j, i}\right) / 2$, the director $n_{i}$ and the rate of change of the director with respect to the background fluid. The latter is written as

$$
\vec{N}=\partial_{t} \vec{n}+(\vec{v} \cdot \nabla) \vec{n}-(\nabla \wedge \vec{v}) \wedge \vec{n} / 2,
$$


where the first two terms represent the substantial derivative of $\vec{n}$ and the third one represents the rotation of the fluid with respect to $\vec{n}$.

In general, the director field $\vec{n}$ can be obtained by imposing the elastic and viscous torques to be in equilibrium. For simplicity, however, we assume that the viscous torque is much smaller than the elastic one, so that the director remains uniform and constant throughout the fluid and $\vec{N}=-(\nabla \wedge \vec{v}) \wedge \vec{n} / 2$. This assumption is known as the small Ericksen number limit (Stark and Ventzki, 2001) and is likely reasonable to model passive PTM experiments, where the thermal energy driving the motion, $k_{B} T$, is extremely low and not expected to alter the nematic configuration. We further assume that the Reynolds number, $R e=\rho U a / \alpha_{4}$, is very small. Typical parameter values in PTM are $\rho \sim 10^{3} \mathrm{~kg} \mathrm{~m}^{-3}, U \sim 10^{-6} \mathrm{~m} \mathrm{~s}^{-1}, a \sim 10^{-6} \mathrm{~m}, \alpha_{4} \sim 10^{-3} \mathrm{~Pa} \mathrm{~s}^{-1}$ and $R e \sim 10^{-6}$, so the low-Reynolds-number simplification is reasonable in this problem. These simplifications make the resulting equations analogous to those describing the dynamics of nematic visco-elastomers (Stenull and Lubensky, 2004) when only the viscous terms are retained in the latter.

It is possible to reduce the number of independent viscosity coefficients from the six that appear in the Leslie-Ericksen constitutive equation (5) to four by introducing the Miesowicz coefficients (Miesowicz, 1936),

$$
\begin{aligned}
& \eta_{a}=\alpha_{4} / 2, \\
& \eta_{b}=\left(\alpha_{3}+\alpha_{4}+\alpha_{6}\right) / 2, \\
& \eta_{c}=\left(-\alpha_{2}+\alpha_{4}+\alpha_{5}\right) / 2,
\end{aligned}
$$

and by using Parodi's relation (Parodi, 1970),

$$
\alpha_{6}=\alpha_{2}+\alpha_{3}+\alpha_{5} .
$$

Under these simplifications and, using a Cartesian coordinate system in which $\vec{n}=(1,0,0)$, the momentum balance equation (4) can be written as

$$
\begin{aligned}
& \partial_{x} p^{\prime}=\eta_{b} \nabla^{2} u+\alpha_{1} \partial_{x x} u+f_{x}, \\
& \partial_{y} p^{\prime}=\eta_{a} \nabla^{2} v+\left(\eta_{c}-\eta_{a}\right) \partial_{x} \omega_{z}+f_{y}, \\
& \partial_{z} p^{\prime}=\eta_{a} \nabla^{2} w-\left(\eta_{c}-\eta_{a}\right) \partial_{x} \omega_{y}+f_{z},
\end{aligned}
$$

where $\vec{r}=(x, y, z)$ is the position vector, $\vec{v}=(u, v, w)$ is the velocity vector, $\vec{\omega}=\left(\omega_{x}, \omega_{y}, \omega_{z}\right)$ is the vorticity vector and $p^{\prime}=p-\alpha_{5} \partial_{x} u$ is a modified pressure. This form of the momentum balance equations is not the most suitable one to solve for the flow around a sphere, but it is included here to facilitate the physical interpretation of the Miesowicz viscosity coefficients. The equations suggest that the two first Miesowicz coefficients, $\eta_{a}$ and $\eta_{b}$, govern the diffusion of the momentum components perpendicular $(\perp)$ and parallel $(\|)$ to $\vec{n}$, respectively. In particular, $\eta_{a}$ is equivalent to the viscosity in an isotropic fluid and the difference $\eta_{b}-\eta_{a}$ indicates the level of anisotropy in the stress-strain relation due to the nematic order of the fluid. Apart from the momentum diffusion Laplacians, equations (6)-(8) have contributions from ||-derivatives of the vorticity. These 
terms represent bending of the fluid with respect to the nematic and are proportional to $\eta_{c}-\eta_{a}$. This second source of anisotropy is perhaps less intuitive than the anisotropy of the stress-strain relation but it can modify significantly the response function of a sphere, as shown in $\$ 4$.

Taking the curl of equations (6)-(8) yields the vorticity equation,

$$
\left[\eta_{a} \nabla^{2}+\left(\eta_{c}-\eta_{a}\right) \partial_{x x}\right] \vec{\omega}+\left[\left(\eta_{b}-\eta_{a}\right) \nabla^{2}+\alpha_{1} \partial_{x x}\right]\{\nabla \wedge[(\vec{u} \cdot \vec{n}) \vec{n}]\}=\overrightarrow{0},
$$

which is analogous to Laplace's vorticity equation for an isotropic, low-Re flow, and where terms in addition to $\nabla^{2} \vec{\omega}$ clearly show the two anisotropy mechanisms mentioned above: the anisotropy in the stress-strain relationship, which is proportional to $\eta_{b}-\eta_{a}$, and the bending resistance of the fluid, which is proportional to $\eta_{c}-\eta_{a}$. Unfortunately, the physical interpretation of $\alpha_{1}$ appears to be less clear. This parameter bears relation to the compressibility of the fluid in the direction of the nematic, as it can be deduced by inspecting the constitutive equation (5). Nevertheless, our results suggest that $\alpha_{1}$ affects the response function of a sphere less than the other viscosity coefficients (see \$4).

\section{Green's function for the flow in an orthotropic fluid}

The response function of a particle in the linear regime can be calculated by using a multipole expansion (Kim and Karrila, 2005, Liron and Barta, 1992 Ladyzhenskaya, 1969). The first step is to calculate the Green's function for the flow. This task is simplified by defining a second modified pressure,

$$
\bar{p}=p^{\prime}+\left(\eta_{c}-\eta_{a}\right) \partial_{x} u=p+\left(\eta_{c}-\eta_{a}-\alpha_{5}\right) \partial_{x} u,
$$

so that equations (6) to (8) become separated in $u, v$ and $w$,

$$
\begin{array}{lr}
\partial_{x} \bar{p}=\left(\eta_{c}+\eta_{b}-\eta_{a}+\alpha_{1}\right) \partial_{x x} u+\eta_{b}\left(\partial_{y y}+\partial_{z z}\right) u+f_{x}, \\
\partial_{y} \bar{p}= & \eta_{c} \partial_{x x} v+\eta_{a}\left(\partial_{y y}+\partial_{z z}\right) v+f_{y}, \\
\partial_{z} \bar{p}= & \eta_{c} \partial_{x x} w+\eta_{a}\left(\partial_{y y}+\partial_{z z}\right) w+f_{z} .
\end{array}
$$

In these equations, the boundary conditions introduced by the sphere are replaced by a point force at the origin before shifting to Fourier space. Because the problem is linear, we assume a solution of the form

$$
\begin{aligned}
& \vec{v}=\frac{\overline{\overline{\mathcal{G}}} \cdot \vec{F}}{8 \pi}=\frac{1}{8 \pi}\left\{\begin{array}{l}
\mathcal{G}_{1 j} F_{j} \\
\mathcal{G}_{2 j} F_{j} \\
\mathcal{G}_{3 j} F_{j}
\end{array}\right\}, \\
& \bar{p}=\frac{\overrightarrow{\mathcal{P}} \cdot \vec{F}}{8 \pi}=\frac{1}{8 \pi} \mathcal{P}_{j} F_{j},
\end{aligned}
$$

where $\mathcal{G}_{i j}$ is the Green's function for the velocity (also called the Oseen tensor) and $\mathcal{P}_{j}$ is the Green's function for the pressure. Solving for the Green's function 
in Fourier space renders (see Appendix $\mathrm{A}$ for more details)

$$
\frac{\widehat{\mathcal{P}}_{j}}{\sqrt{8 / \pi}}=i k_{j}\left[\frac{1-\delta_{1 j}}{k^{2}}+\frac{\frac{1-\delta_{1 j}}{k^{2}}\left(\alpha_{1} k_{1}^{4}-\eta_{a} k^{4}\right)+\eta_{a} k^{2}+\left(\eta_{b}-\eta_{a}\right) k_{1}^{2}+\left(\eta_{c}-\eta_{b}\right) k_{1}^{2} \delta_{1 j}}{\alpha_{1} k_{1}^{2}\left(k_{2}^{2}+k_{3}^{2}\right)+\eta_{b} k^{4}+\left(\eta_{c}-\eta_{b}\right) k_{1}^{2} k^{2}}\right],
$$

and

$$
\begin{aligned}
& \frac{\widehat{\mathcal{G}}_{1 j}}{\sqrt{8 / \pi}}=\frac{\delta_{1 j} k^{2}-k_{1} k_{j}}{\alpha_{1} k_{1}^{2}\left(k_{2}^{2}+k_{3}^{2}\right)+\eta_{b} k^{4}+\left(\eta_{c}-\eta_{b}\right) k_{1}^{2} k^{2}} \\
& \frac{\widehat{\mathcal{G}}_{2 j}}{\sqrt{8 / \pi}}=\left.\frac{\delta_{2 j}}{\left(\eta_{c}-\eta_{a}\right) k_{1}^{2}+\eta_{a} k^{2}}-k_{2} k_{j} \frac{\left(1-\delta_{1 j}\right) \frac{\alpha_{1} k_{1}^{2}+\left(\eta_{b}-\eta_{a}\right) k^{2}}{\left(\eta_{c}-\eta_{a}\right) k_{1}^{2}+\eta_{a} k^{2}}+1}{\alpha_{1} k_{1}^{2}\left(k_{2}^{2}+k_{3}^{2}\right)+\eta_{b} k^{4}+\left(\eta_{c}-\eta_{b}\right) k_{1}^{2} k^{2}} 6\right) \\
& \frac{\widehat{\mathcal{G}}_{3 j}}{\sqrt{8 / \pi}}=\frac{\delta_{3 j}}{\left(\eta_{c}-\eta_{a}\right) k_{1}^{2}+\eta_{a} k^{2}}-k_{3} k_{j} \frac{\left(1-\delta_{1 j}\right) \frac{\alpha_{1} k_{1}^{2}+\left(\eta_{b}-\eta_{a}\right) k^{2}}{\left(\eta_{c}-\eta_{a}\right) k_{1}^{2}+\eta_{a} k^{2}}+1}{\alpha_{1} k_{1}^{2}\left(k_{2}^{2}+k_{3}^{2}\right)+\eta_{b} k^{4}+\left(\eta_{c}-\eta_{b}\right) k_{1}^{2} k^{2}}
\end{aligned}
$$

In this formulation, $\vec{k}=\left(k_{1}, k_{2}, k_{3}\right)=\left(k_{x}, k_{y}, k_{z}\right)$ is the wavenumber vector in the Fourier domain, $\delta_{i j}$ is the Dirac delta function and $i$ the imaginary unit. In the isotropic limit case where $\eta_{a}=\eta_{b}=\eta_{c}=\eta$ and $\alpha_{1}=0$, we recover the Green's functions for isotropic fluids as expected.

\section{Response Function of a spherical particle in a Nematic Fluid}

In the low-Reynolds-number limit, the velocity of a particle moving in an orthotropic fluid and the drag force exerted on it are related as

$$
\vec{F}=\overline{\bar{\zeta}} \cdot \vec{v}(\vec{x}=\overrightarrow{0})=\overline{\bar{\zeta}} \cdot \vec{v}_{0}
$$

where $\overline{\bar{\zeta}}$ is a tensorial response function, also known as hydrodynamic resistance (Kim and Karrila, 2005). The aim of this section is to obtain analytical expressions for the different elements of $\overline{\bar{\zeta}}$. Given the singularity at the origin, the response function cannot be directly calculated from equation 13 but it can be obtained by regularizing the solution and assuming that the force applied by the sphere is not a point force but a compact force distribution $\vec{F}(\vec{x})$ (Levine and Lubensky, 2001; Liron and Barta, 1992). The velocity at the origin can be obtained from the Fourier transform of the Green's function as

$$
\vec{v}_{0}=\frac{1}{(2 \pi)^{3 / 2}} \iiint \frac{\widehat{\overline{\mathcal{G}}}}{8 \pi} \cdot \overrightarrow{\widehat{F}}(\vec{k}) d^{3} k=\overline{\bar{\gamma}} \cdot \vec{F},
$$

where $\overrightarrow{\widehat{F}}(\vec{k})=\vec{F} \widehat{\mathcal{F}}(\vec{k})$ is the Fourier transform of $\vec{F}(\vec{x})$, the function $\widehat{\mathcal{F}}(\vec{k})$ is a regularization kernel that localized the drag force in physical and/or Fourier space, and $\overline{\bar{\gamma}}$ is the hydrodynamic mobility. Note that equation 19 ) is just the 
inverse Fourier transform of $\overrightarrow{\widehat{v}}(\vec{k})$ particularized at the origin, and that equations 18)-190 imply that $\overline{\bar{\zeta}}=\overline{\bar{\gamma}}^{-1}$.

Levine and Lubensky (2001) propose a volume localization approach to simplify the calculation of $\overline{\bar{\gamma}}$ in the isotropic case by considering that the particle radius limits the spectrum of allowed fluctuations in the flow field. Hence, they only consider wavenumbers smaller than $k_{\max }=\pi / 2 a$. This approximation is written as

$$
\widehat{\mathcal{F}}(k)=H\left(\frac{\pi}{2 a}-k\right),
$$

where $H$ is the Heaviside function. It leads to the following expression for the inverse response function tensor

$$
\gamma_{i j}=\frac{1}{4 \sqrt{2 \pi} a} \int_{\theta=0}^{\theta=\pi} \sin \theta\left(\int_{\varphi=0}^{\varphi=2 \pi} \frac{k^{2} \widehat{\mathcal{G}}_{i j}}{8 \pi} d \varphi\right) d \theta,
$$

where $\theta$ and $\varphi$ are the inclination and azimuth angles in spherical coordinates, respectively. Note that $\widehat{\mathcal{G}}_{i j}$ is inversely proportional to $k^{2}$, so that the whole expression is independent of $k$. While it holds a clear physical meaning, the sharp Fourier cut-off of the Heaviside regularization function leads to a nonlocalized force distribution in physical space and a velocity field with Gibbs oscillations in the radial direction. For this reason, the Gaussian regularization function

$$
\widehat{\mathcal{F}}(k)=e^{-a^{2} k^{2} / \pi}
$$

is preferred in this study. This choice yields the same equation for $\gamma_{i j}$ and has the advantage of providing a localized Gaussian force distribution and a smooth velocity field in physical space (see \$6). This approach is analogous to the regularized Stokeslet method (Beale and Lai, 2001, Cortez, 2001).

Owing to the symmetry of the Green's function (15)-(17), we obtain that $\gamma_{i j}=0$ for $i \neq j$, and both $\overline{\bar{\gamma}}$ and $\overline{\bar{\zeta}}$ are diagonal tensors. In particular,

$$
\vec{F}=\left[\begin{array}{ccc}
\zeta_{\|} & 0 & 0 \\
0 & \zeta_{\perp} & 0 \\
0 & 0 & \zeta_{\perp}
\end{array}\right] \cdot \vec{v}_{0},
$$

where $\zeta_{\|}$is the response function in the direction parallel to the nematic and $\zeta_{\perp}$ is the response function in all transverse directions. These principal values can be determined independently by measuring the drag force of a particle in the directions parallel and perpendicular to $\vec{n}$. For any other direction of motion, the drag force acting on a particle is not parallel to $\vec{v}_{0}$. In general, if $\vec{v}_{0}$ forms an angle $\beta_{v}$ with $\vec{n}$, the angle between the drag force vector and the nematic director is $\beta_{F}=\arctan \left[\tan \left(\beta_{v}\right) \zeta_{\perp} / \zeta_{\|}\right]$(see figure 1).

Armed with some tenacity, one can find an explicit analytical solution to the integrals in equation 20, leading to the following expressions for the diagonal 
components of the response function,

$$
\begin{aligned}
& \zeta_{\|}=\frac{1}{\gamma_{11}}=\frac{8 \pi a \eta_{b} B(\vec{\eta})}{D_{+}(\vec{\eta}) \frac{\arctan \left[C_{+}(\vec{\eta})\right]}{C_{+}(\vec{\eta})}-D_{-}(\vec{\eta}) \frac{\arctan \left[C_{-}(\vec{\eta})\right]}{C_{-}(\vec{\eta})}} \\
& \zeta_{\perp}=\frac{1}{\gamma_{22}}=\frac{1}{\gamma_{33}}=\frac{8 \pi a \eta_{a}\left(\frac{\eta_{b}}{\alpha_{1}}\right)^{2} C_{-}(\vec{\eta})^{8} \frac{E_{+}(\vec{\eta})}{E_{-}(\vec{\eta})}}{\frac{\arctan \left(\sqrt{\eta_{c} / \eta_{a}-1}\right)}{\sqrt{\eta_{c} / \eta_{a}-1}}+\frac{1}{B(\vec{\eta})} \frac{\eta_{a}}{\eta_{b}}\left[\frac{\arctan \left[C_{-}(\vec{\eta})\right]}{C_{-}(\vec{\eta})}-\frac{\arctan \left[C_{+}(\vec{\eta})\right]}{C_{+}(\vec{\eta})}\right]}
\end{aligned}
$$

where $B(\vec{\eta}), C_{ \pm}(\vec{\eta}), D_{ \pm}(\vec{\eta})$ and $E_{ \pm}(\vec{\eta})$ are non-dimensional functions of the viscosity vector $\vec{\eta}=\left(\eta_{a}, \eta_{b}, \eta_{c}, \alpha_{1}\right)$. These functions are given in Appendix B Equations (23) and (24) have a weak singularity at $\alpha_{1}=0$ that can be removed by taking the limit $\alpha_{1} \rightarrow 0$, leading to

$$
\begin{aligned}
\zeta_{\|, \alpha_{1}=0} & =\frac{4 \pi a\left(\eta_{c}-\eta_{b}\right)}{\frac{\eta_{c}}{\eta_{b}} \frac{\arctan \left(\sqrt{\eta_{c} / \eta_{b}-1}\right)}{\sqrt{\eta_{c} / \eta_{b}-1}}-1}, \\
\zeta_{\perp, \alpha_{1}=0} & =\frac{8 \pi a\left(\eta_{c}-\eta_{b}\right)}{1-\frac{\arctan \left(\sqrt{\eta_{c} / \eta_{b}-1}\right)}{\sqrt{\eta_{c} / \eta_{b}-1}}+\frac{\eta_{c}-\eta_{b}}{\eta_{a}} \frac{\arctan \left(\sqrt{\eta_{c} / \eta_{a}-1}\right)}{\sqrt{\eta_{c} / \eta_{a}-1}}} .
\end{aligned}
$$

The isotropy point is of particular interest because diluted networks should not be too far from it. Taylor expanding equations (23) and 24) around the point $\alpha_{1}=0, \eta_{a}=\eta_{b}=\eta_{c}=\eta$, and keeping only the first order term we obtain

$$
\begin{aligned}
\frac{\zeta_{\|}}{6 \pi a \eta} & \approx 1+\frac{4}{35} \frac{\alpha_{1}}{\eta}+\frac{4}{5}\left(\frac{\eta_{b}}{\eta}-1\right)+\frac{1}{5}\left(\frac{\eta_{c}}{\eta}-1\right), \\
\frac{\zeta_{\perp}}{6 \pi a \eta} & \approx 1+\frac{3}{70} \frac{\alpha_{1}}{\eta}+\frac{1}{2}\left(\frac{\eta_{a}}{\eta}-1\right)+\frac{1}{10}\left(\frac{\eta_{b}}{\eta}-1\right)+\frac{2}{5}\left(\frac{\eta_{c}}{\eta}-1\right) .
\end{aligned}
$$

This Taylor expansion is consistent with previous results by Pokrovskii and Tskhai (1986), who studied the motion of a particle in a weakly anisotropic fluid with $\eta_{b}=\eta_{c}$.

In general, both components of the response function increase with increasing the viscosity coefficients but there are several aspects of this dependence that deserve especial attention. Figure 2 displays the response function in the nematic direction, $\zeta_{\|}=\zeta_{11}$. Interestingly, this component is independent of the first Miesowicz viscosity $\eta_{a}$, as shown in figure 2(a) for $\alpha_{1}=0$, and in agreement with Kneppe et al. (1991). This degeneracy can be explained by realizing that the flow elicited by a sphere that translates parallel to the nematic director is axially symmetric. Working in polar cylindrical coordinates $(r, \theta, x)$ and taking into account this symmetry, the momentum balance equations (6)-(8) can be rewritten as

$$
\begin{aligned}
& \partial_{x} p^{\prime}=\eta_{b} \nabla^{2} u_{x}+\alpha_{1} \partial_{x x} u_{x}, \\
& \partial_{r} p^{\prime}=\eta_{c}\left(\nabla^{2} u_{r}-u_{r} / r^{2}\right)-\left(\eta_{c}-\eta_{a}\right) \partial_{r}(\nabla \cdot \vec{v}),
\end{aligned}
$$



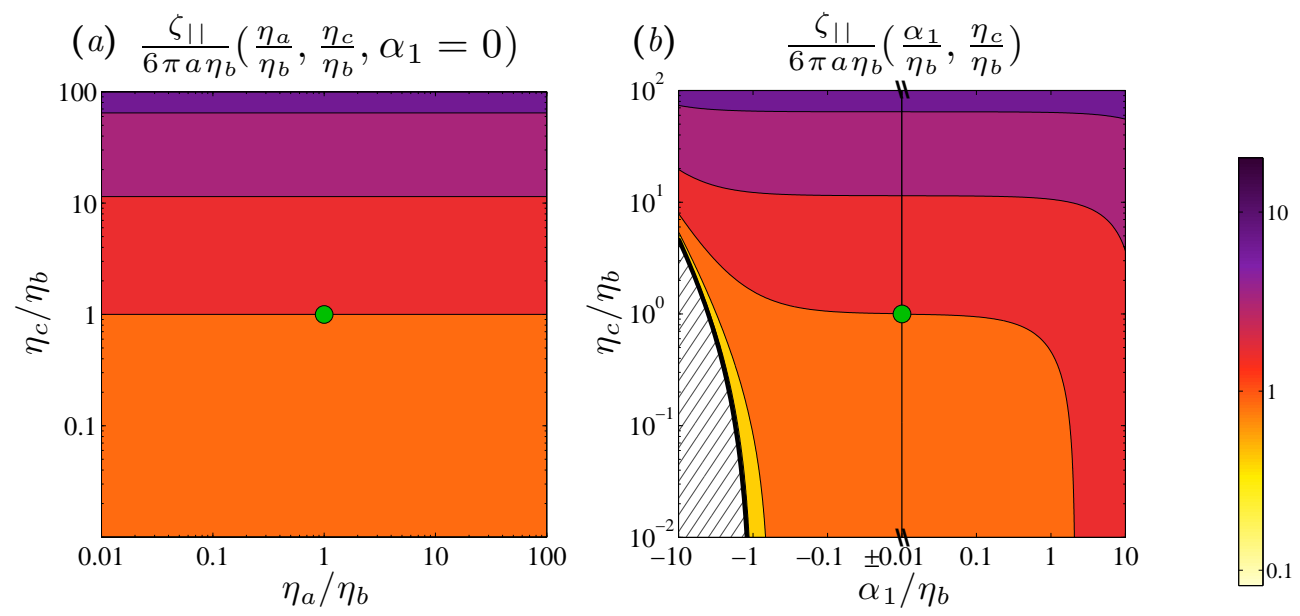

Figure 2: Contour maps of the response function in the nematic direction, $\zeta_{\|}$, obtained from eq. (23): $(a), \zeta_{\|}\left(\eta_{a} / \eta_{b}, \eta_{c} / \eta_{b}\right)$ for $\alpha_{1}=0$, showing that this response function is independent of $\eta_{a} ;(b), \zeta_{\|}\left(\alpha_{1} / \eta_{b}, \eta_{c} / \eta_{b}\right)$. Note that the $x$ axis is broken between $\alpha_{1}=-0.01 \eta_{b}$ and $\alpha_{1}=0.01 \eta_{b}$. The thick black curve indicates the locus where $\zeta_{\|}=0$. The hatched region to the left of this curve yields unphysical, complex-valued results for the response function. In both panels, the response functions have been normalized with $6 \pi a \eta_{b}$. The green circle represents the isotropic case with $\eta_{a}=\eta_{b}=\eta_{c}$ and $\alpha_{1}=0$.
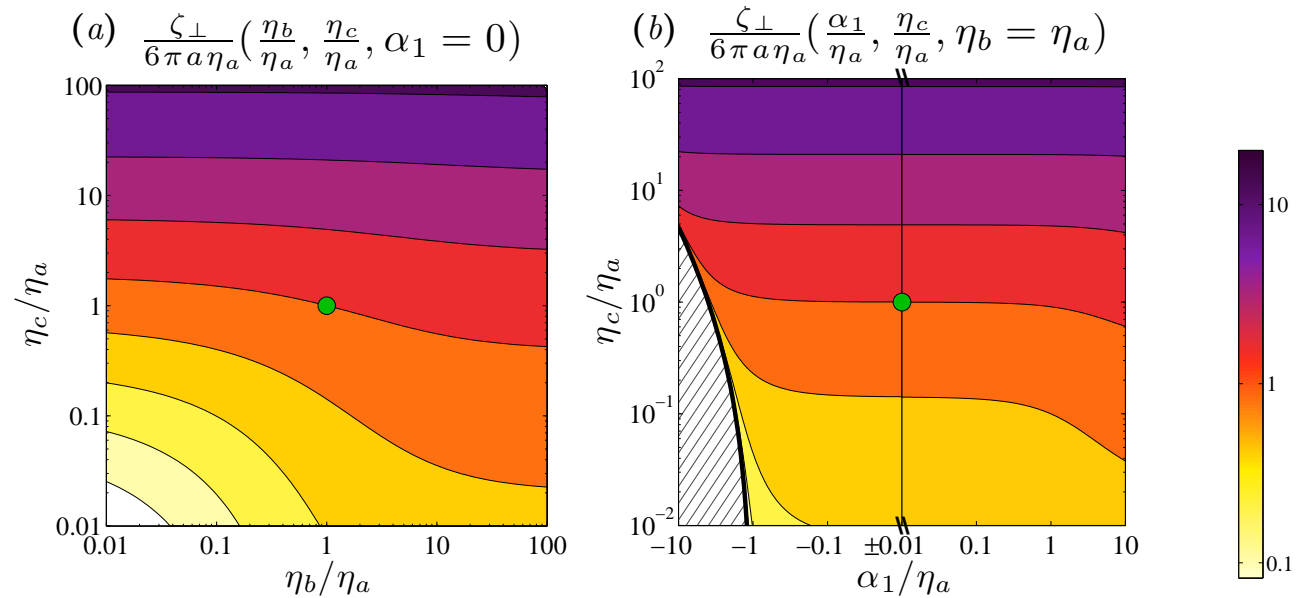

Figure 3: Contour maps of the response function perpendicular to the nematic, $\zeta_{\perp}$, obtained from equation (24): $(a), \zeta_{\perp}\left(\eta_{b} / \eta_{a}, \eta_{c} / \eta_{a}\right)$ for $\alpha_{1}=0$; (b), $\zeta_{\perp}\left(\eta_{b} / \eta_{a}, \alpha_{1} / \eta_{a}\right)$ for $\eta_{b}=\eta_{a}$. Note that the $x$-axis is broken between $\alpha_{1}=-0.01 \eta_{a}$ and $\alpha_{1}=0.01 \eta_{a}$. The thick black curve indicates the locus where $\zeta_{\perp}=0$. The region to the left of this curve yields unphysical, complex-valued results for the response function. In both panels, the response functions have been normalized with $6 \pi a \eta_{a}$. The green circle represents the isotropy point, $\eta_{a}=\eta_{b}=\eta_{c}$ and $\alpha_{1}=0$. 
where $\eta_{c}-\eta_{a}$ acts as a dilatational viscosity in the radial direction that disappears when the velocity field is divergence-free. It is recognized that the resulting divergence-free, axially symmetric velocity field has a stream function but this function is not pursued here for the sake of consistency with the perpendicular problem, which is not axially symmetric. Note also that the Green's function derived in $\$ 3$ can be easily extended to networks with significant volume fractions, where network compressibility leads to $\nabla \cdot \vec{v} \neq 0$, and there is no stream function.

Figure 2(b) depicts the dependence of the parallel response function on the remaining three viscosity coefficients by displaying $\zeta_{\|} /\left(a \eta_{b}\right)$ as a function of $\alpha_{1} / \eta_{b}$ and $\eta_{c} / \eta_{b}$. This non-dimensionalization is chosen because the viscous shear stresses in the $\|$ direction are proportional to $\eta_{b}$ (see equation 6). In this scaling, the parallel response function shows a moderate dependence on the ratio $\eta_{c} / \eta_{b}$. In fact, the limiting values of $\zeta_{\|}\left(\alpha_{1}=0\right)$ for $\eta_{c} / \eta_{b} \rightarrow 0, \infty$ are $4 \pi a \eta_{b}$ and $8 a \sqrt{\eta_{b} \eta_{c}}$, respectively, only differing by a factor of order $\left(\eta_{c} / \eta_{b}\right)^{1 / 2}$.

The dependence of the parallel response function on $\alpha_{1}$ is even weaker, especially in the case $\eta_{c} \gg \eta_{b}$, which is dominated by the viscous stresses in the $\perp$ directions. Near the isotropy point, the parallel response function is relatively insensitive to $\alpha_{1}$, consistent with the small factor multiplying this coefficient in the Taylor expansion of equation (27). Figure 2(b) also considers $\alpha_{1}<0$ as this viscosity coefficient has been reported to be negative in some nematic liquid crystals (Kneppe and Schneider, 1981). Our analysis indicates that lowering $\alpha_{1}$ below zero causes the response function to decrease. This decrease is moderate everywhere but near the curve

$$
\frac{\eta_{c}}{\eta_{b}}=1-\frac{\alpha_{1}}{\eta_{b}}-2 \sqrt{\frac{-\alpha_{1}}{\eta_{b}}}, \quad \alpha_{1}<-\eta_{b},
$$

where the function $B(\vec{\eta})$ that multiplies the right-hand side of equation (23) becomes zero and so does the parallel response function (see Appendix B). The region to the left of this curve in the $\left(\alpha_{1} / \eta_{b}, \eta_{c} / \eta_{b}\right)$ plane produces complex values of $\zeta_{\|}$and, thus, is physically impossible.

The transverse response function, $\zeta_{\perp}=\zeta_{22}$ (equation 24), has a more complicated dependence on the viscosity coefficients than its parallel counterpart because the flow elicited by $\vec{v}_{0}$ is not axially symmetric. In order to analyze this dependence, it is convenient to plot $\zeta_{\perp} /\left(a \eta_{a}\right)$ as a function of $\eta_{b} / \eta_{a}, \eta_{c} / \eta_{a}$ and $\alpha_{1} / \eta_{a}$. This representation allows us to evaluate the relative importance of the different sources of anisotropy in the Leslie-Ericksen constitutive equation (5), namely bending of the fluid with respect to the nematic and anisotropy in the stress-strain relationship.

Figure 3( $a)$ displays the transverse response function in the case $\alpha_{1}=0$. The plot reveals that $\zeta_{\perp} /\left(a \eta_{a}\right)$ is more sensitive to $\eta_{c} / \eta_{a}$ than to $\eta_{b} / \eta_{a}$. This is especially true in the limit $\eta_{c} \gg \eta_{a}$, in which the bending stresses become dominant in the equations of motion, and the response function has a concise limit that is independent of the second Miesowicz viscosity,

$$
\zeta_{\perp} \rightarrow 16 a \eta_{a} \sqrt{\eta_{c} / \eta_{a}} .
$$


The response function also becomes independent of $\eta_{b}$ in the limit $\eta_{b} \gg \eta_{a}$, in which the anisotropic momentum diffusivity dominates the flow dynamics, and

$$
\zeta_{\perp} \rightarrow 8 \pi a \eta_{a} \sqrt{\eta_{c} / \eta_{a}-1} / \arctan \left(\sqrt{\eta_{c} / \eta_{a}-1}\right) .
$$

The $\alpha_{1}$-dependence of $\zeta_{\perp}$ is similar to that of $\zeta_{\|}$, as shown in figure $3(b)$ for the case $\eta_{a}=\eta_{b}$. The transverse response function is fairly insensitive to $\alpha_{1}$ for the most part of the parameter space, particularly near the isotropy point and for large $\eta_{c} / \eta_{a}$. Much like the $\|$ case, the effect of $\alpha_{1}$ only becomes strong near the curve defined by equation (31), where the transverse response function is zero. In the scaling used to plot figure $3(b)$, this curve has an asymptote at $\left(\alpha_{1} / \eta_{a}, \eta_{c} / \eta_{a}\right)=\left(-\eta_{b} / \eta_{a}, 0\right)$. Thus, as $\eta_{b}$ increases, the asymptote moves towards larger negative values of $\alpha_{1} / \eta_{a}$ and the influence of $\alpha_{1}$ becomes less important.

\subsection{Pseudo-isotropic conditions}

The analysis of the equations of motion (6)-(9) suggests that the anisotropy in the Leslie-Ericksen constitutive relation (5) may influence the response function by means of two separate mechanisms. One is the anisotropy of the stress-strain relationship, which is proportional to the viscosity difference $\eta_{b}-\eta_{a}$, while the other is the resistance to the bending of the fluid, which is proportional to the viscosity difference $\eta_{c}-\eta_{a}$. The aim of this section is to dissect the effects of the two anisotropy mechanisms. We first study the response function in a rotationally pseudo-isotropic fluid where anisotropy comes exclusively from the anisotropic momentum diffusivity, and which corresponds to the horizontal line $\eta_{c}=\eta_{a}$ in figures 2 $(a)$ and 3( $\left.a\right)$. We then consider a strain pseudo-isotropic fluid, where the anisotropy comes solely from the resistance to bending of the fluid with respect to the nematic, and which corresponds to the vertical line $\eta_{b}=\eta_{a}$ in figures $2(a)$ and $3(a)$.

The response functions derived in this section also provide with distinct two-viscosity benchmarks to test the accuracy of existing PTM methods when applied to anisotropic fluids (see \$5). This assessment needs to be performed on two-viscosity fluids as only two of the principal components of the response functions are independent in a nematic fluid (see equation 22). This limitation can be potentially circumvented by considering two-point response functions but such effort is beyond the scope of this paper.

An aspect of particular interest that is evaluated on pseudo-isotropic fluids is the accuracy of the directional effective viscosity approximation. Based on the principal components of the response function, one can define two effective directional viscosities $\eta_{\|, \perp}^{\text {eff }}=\zeta_{\|, \perp} /(6 \pi a)$, similar to Stark and Ventzki (2001). This phenomenological approach has been previously used by experimentalists to characterise different types of nematic fluids (Loudet et al. 2004: Hasnain and Donald, 2006, Rogers et al. 2008, del Álamo et al., 2008; He et al., 2008) but it has not been justified yet. 
(a)

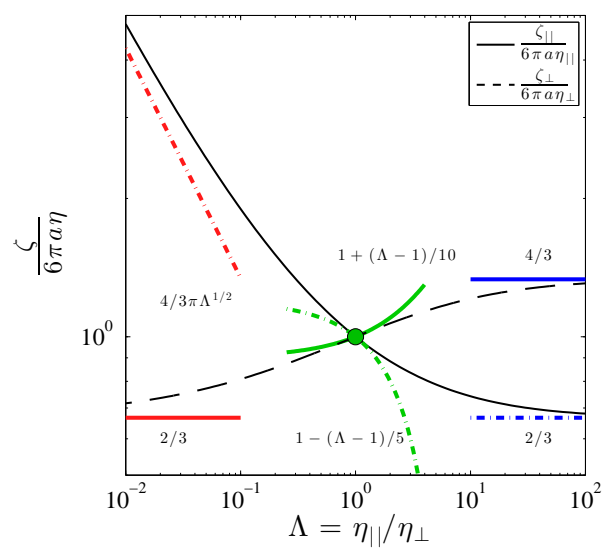

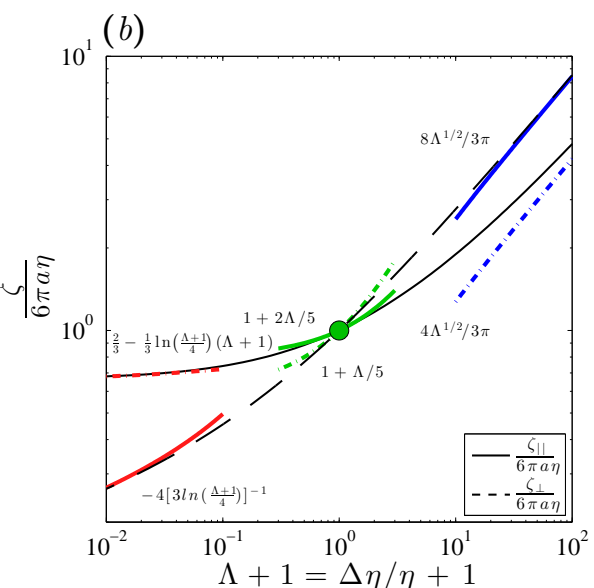

Figure 4: Principal components of the response function $(a)$ in a rotationally pseudo-isotropic fluid, $\alpha_{1}=0, \eta_{b}=\eta_{\|}$and $\eta_{a}=\eta_{c}=\eta_{\perp}$, represented as a function of the viscosity ratio $\Lambda=\eta_{\|} / \eta_{\perp}$ and (b) in a strain pseudo-isotropic fluid $\alpha_{1}=0, \eta_{a}=\eta_{b}=\eta$ and $\eta_{c}=\Delta \eta+\eta$, represented as a function of the viscosity ratio $\Lambda=\Delta \eta / \eta:-, \zeta_{\|} / 6 \pi a \eta_{\|}:---, \zeta_{\perp} / 6 \pi a \eta_{\perp}$. The green circle marks the isotropy point: $(a) \Lambda=1$ and $(b) \Lambda=0$. The thick color segments mark the asymptotic behavior of the response function for $(a) \Lambda \ll 1$ (red), $\Lambda \approx 1$ (green) and $\Lambda \gg 1$ (blue), and (b) $\Lambda \rightarrow-1$ (red), $\Lambda \approx 0$ (green) and $\Lambda \gg 1$ (blue).

\subsubsection{Rotationally Pseudo-Isotropic Fluid $\left(\eta_{a}=\eta_{c}\right)$}

Consider a fluid where $\alpha_{1}$ is very small and $\alpha_{2}=\alpha_{5}$. Under these conditions, the last term in the transverse components of the momentum balance equation (equations 7 8 disappears, yielding the rotationally isotropic pseudoStokes problem

$$
\nabla p^{\prime}=\left[\begin{array}{rcc}
\eta_{\|} & 0 & 0 \\
0 & \eta_{\perp} & 0 \\
0 & 0 & \eta_{\perp}
\end{array}\right] \cdot \nabla^{2} \vec{v},
$$

where $\eta_{\|}=\eta_{b}$ denotes the viscosity parallel to the director and $\eta_{\perp}=\eta_{a}$ is the viscosity in the transverse directions. In this case, the directionality of the problem is solely due to the anisotropic diffusion of momentum along different directions (i.e. anisotropy in the stress-strain relationship).

Taking the limit $\eta_{a} \rightarrow \eta_{c}$ on equations (23)-24), and introducing the anisotropy 
ratio $\Lambda=\eta_{\|} / \eta_{\perp}$, we find that

$$
\begin{aligned}
\frac{\zeta_{\|}}{6 \pi a \eta_{\|}}= & \frac{\frac{2}{3}(1-\Lambda)}{-\Lambda+\frac{\arctan (\sqrt{1 / \Lambda-1})}{\sqrt{1 / \Lambda-1}}}, \\
\frac{\zeta_{\perp}}{6 \pi a \eta_{\perp}}= & \frac{\frac{4}{3}(1-\Lambda)}{-\Lambda+2-\frac{\arctan (\sqrt{1 / \Lambda-1})}{\sqrt{1 / \Lambda-1}}} .
\end{aligned}
$$

These response functions are plotted in figure 4 $(a)$ together with their tangent lines at the isotropy point $\Lambda=1$ and their asymptotes for highly anisotropic conditions $(\Lambda \gg 1$ and $\Lambda \ll 1)$. This figure shows $1 \mathrm{D}$ cuts of figures $2(a)$ and 3(a) along the horizontal line $\eta_{c}=\eta_{a}$.

The behavior of the $\|$ and $\perp$ response functions near the isotropy point is given by their Taylor expansion

$$
\begin{aligned}
& \frac{\zeta_{\|}}{6 \pi a \eta_{\|}}=1-\frac{1}{5}(\Lambda-1)+\frac{27}{175}(\Lambda-1)^{2}+O\left[(\Lambda-1)^{3}\right], \\
& \frac{\zeta_{\perp}}{6 \pi a \eta_{\perp}}=1+\frac{1}{10}(\Lambda-1)-\frac{33}{700}(\Lambda-1)^{2}+O\left[(\Lambda-1)^{3}\right],
\end{aligned}
$$

where the leading-order terms correspond to the expected isotropic result $\zeta=$ $6 \pi a \eta$, and the higher-order terms are the corrections introduced by the anisotropy of the fluid. The numerical values of the coefficients in these expansions indicate that the anisotropic corrections are weaker in the transverse response function than in the parallel one, consistent with the weaker variation of $\zeta_{\perp}$ observed in figure $4(a)$.

In order to determine whether the effects of anisotropy are bounded in a rotationally pseudo-isotropic fluid, we consider the asymptotic behavior of the response function for $\Lambda \gg 1$, where

$$
\frac{\zeta_{\|}}{6 \pi a \eta_{\|}} \simeq \frac{2}{3}, \quad \frac{\zeta_{\perp}}{6 \pi a \eta_{\perp}} \simeq \frac{4}{3} .
$$

Interestingly, these expressions are equivalent to those of a prolate ellipsoid moving along its principal axes in an isotropic liquid (Kim and Karrila, 2005). It is important to note that the two components of the response function are decoupled from each other, so that increasing $\eta_{\|}$does not affect $\zeta_{\perp}$ and vice versa. Thus, the ratio of effective diffusivities becomes proportional to the ratio of actual viscosities of the fluid for $\Lambda \gg 1$,

$$
\frac{\eta_{\|}^{\mathrm{eff}}}{\eta_{\perp}^{\mathrm{eff}}}=\frac{\zeta_{\|}}{\zeta_{\perp}} \simeq \frac{1}{2} \frac{\eta_{\|}}{\eta_{\perp}}
$$

although these ratios differ by a factor of two. 
The second asymptotic limit is $\Lambda \ll 1$, which results in

$$
\frac{\zeta_{\|}}{6 \pi a \eta_{\|}} \simeq \frac{4}{3 \pi \sqrt{\Lambda}}, \quad \frac{\zeta_{\perp}}{6 \pi a \eta_{\perp}} \simeq \frac{2}{3}
$$

It can be seen that, in this limit, the $\|$ component of the response function not only depends on $\eta_{\| \mid}$but also on $\eta_{\perp}$, so that the ratio of effective diffusivities,

$$
\frac{\eta_{\|}^{\mathrm{eff}}}{\eta_{\perp}^{\mathrm{eff}}}=\frac{\zeta_{\|}}{\zeta_{\perp}} \simeq \frac{2}{\pi} \sqrt{\frac{\eta_{\|}}{\eta_{\perp}}} \gg \frac{\eta_{\|}}{\eta_{\perp}},
$$

becomes a rather poor measure of the ratio of actual viscosities of the fluid.

\subsubsection{Strain Pseudo-Isotropic Fluid $\left(\eta_{a}=\eta_{b}\right)$}

Consider a fluid where $\alpha_{1}$ and $\alpha_{3}$ are very small and $\alpha_{2}=-\alpha_{5}$. Under these conditions, the equations of motion (6)-(8) become

$$
\nabla p^{\prime}=\eta \nabla^{2} \vec{v}+\Delta \eta \partial_{\|}(\vec{\omega} \times \vec{n}),
$$

where $\eta=\eta_{a}$ and $\Delta \eta=\eta_{c}-\eta_{a}$. The Laplacian term on the right hand side of equation (35) can be seen as coming from isotropic momentum diffusion with viscosity $\eta$. On the other hand, the last term in the equation represents the resistance of the fluid to bending with respect to the nematic, and this is the only source of anisotropy in the strain pseudo-isotropic condition. The coefficient $\Delta \eta$ can be interpreted as a rotational viscosity.

Taking the limit $\eta_{b} \rightarrow \eta_{a}$ on equations (23)-(24), and introducing the anisotropy ratio $\Lambda=\Delta \eta / \eta$, we find that

$$
\begin{aligned}
& \frac{\zeta_{\|}}{6 \pi a \eta}=\frac{\frac{2}{3} \Lambda}{(\Lambda+1) \frac{\arctan (\sqrt{\Lambda})}{\sqrt{\Lambda}}-1}, \\
& \frac{\zeta_{\perp}}{6 \pi a \eta}=\frac{\frac{4}{3} \Lambda}{(\Lambda-1) \frac{\arctan (\sqrt{\Lambda})}{\sqrt{\Lambda}}+1} .
\end{aligned}
$$

Note that, in this case, the anisotropy ratio ranges between $\Lambda=-1\left(\eta_{c} \ll \eta_{a}\right)$ and $\Lambda=\infty\left(\eta_{c} \gg \eta_{a}\right)$, and the isotropy point corresponds to $\Lambda=0$.

Similar to figure $4(a)$, the parallel and perpendicular response functions for a strain pseudo-isotropic fluid are plotted in figure $4(b)$, along with their tangent lines at the isotropy point and their asymptotes for highly anisotropic conditions. In this case, the plotted curves are one-dimensional sections of figures $2(a)$ and $3(a)$ along the vertical line $\eta_{a}=\eta_{b}$.

The behavior of the response function near the isotropy point is given by its Taylor expansion,

$$
\begin{aligned}
& \frac{\zeta_{\|}}{6 \pi a \eta}=1+\frac{1}{5} \Lambda-\frac{8}{175} \Lambda^{2}+O\left[\Lambda^{3}\right], \\
& \frac{\zeta_{\perp}}{6 \pi a \eta}=1+\frac{2}{5} \Lambda-\frac{17}{175} \Lambda^{2}+O\left[\Lambda^{3}\right] .
\end{aligned}
$$


which shows that, in contrast to the rotationally pseudo-isotropic condition, the anisotropy corrections are stronger in the transverse direction than in the parallel direction. This result is consistent with the intuitive notion that particle motion in the $\perp$ direction bends the nematic more than particle motion in the || direction.

In the limit $\Lambda \gg 1$, the asymptotic behavior of the response function is

$$
\frac{\zeta_{\|}}{6 \pi a \eta} \simeq \frac{4}{3 \pi} \sqrt{\Lambda}, \quad \frac{\zeta_{\perp}}{6 \pi a \eta} \simeq \frac{8}{3 \pi} \sqrt{\Lambda},
$$

whereas, in the limit $\Lambda \rightarrow-1$, we have

$$
\begin{aligned}
\frac{\zeta_{\|}}{6 \pi a \eta} & \simeq \frac{2}{3}-\frac{2}{3}\left[1+\frac{1}{2} \ln \left(\frac{\Lambda+1}{4}\right)\right](\Lambda+1), \\
\frac{\zeta_{\perp}}{6 \pi a \eta} & \simeq-\frac{4}{3} \frac{1}{\ln \left(\frac{\Lambda+1}{4}\right)+1} .
\end{aligned}
$$

Comparing these asymptotic responses with those in figure $4(a)$ reveals that the effects of anisotropy in the stress-strain relationship are fundamentally different from those arising from nematic bending. In rotationally pseudo-isotropic fluids, the response function becomes independent of the anisotropy ratio for high levels of anisotropy, leading to horizontal asymptotes in figure $4(a)$, and rendering upper bounds to the effect of anisotropy. The only exception to this behavior is $\zeta_{\|}\left(\eta_{\|} \ll \eta_{\perp}\right)$, which has a square-root dependence on $\eta_{\perp}$ due to the fact that the flow is still infinitesimally deflected in the $\perp$ direction by the sphere in this limit, as will be shown in 8.1 .

In contrast, the strain pseudo-isotropic response function remains dependent on both $\eta$ and $\Delta \eta$ even at high levels of anisotropy, suggesting a strong interaction between the anisotropic stresses caused by bending and the diffusion of momentum by strain. These two mechanisms only appear to decouple from each other for $\zeta_{\|}(\Delta \eta \rightarrow-\eta)$, which is consistent with the independence of the parallel response with respect to the first Miesowicz coefficient (figure 2a), as $\Delta \eta=-\eta_{a}$ in this limit. These results inevitably call for caution in employing the concept of effective directional viscosities. This notion may be qualitatively useful in rotationally pseudo-isotropic fluids where the effect of anisotropy can be partially separated in different directions. However, its usefulness becomes limited in fluids with resistance to bending, where the anisotropy appears to act in all directions concurrently and in a non-trivial manner.

\subsection{Comparison with numerical results}

This section uses data from existing numerical simulations to validate the analytical procedures employed to determine $\overline{\bar{\zeta}}$. Owing to the relatively high number of different viscosities involved in the problem, it is difficult to find simulation studies that cover a significant part of the parameter space. The most comprehensive set of simulations is Heuer et al. (1992), who compute the steady 
(a)
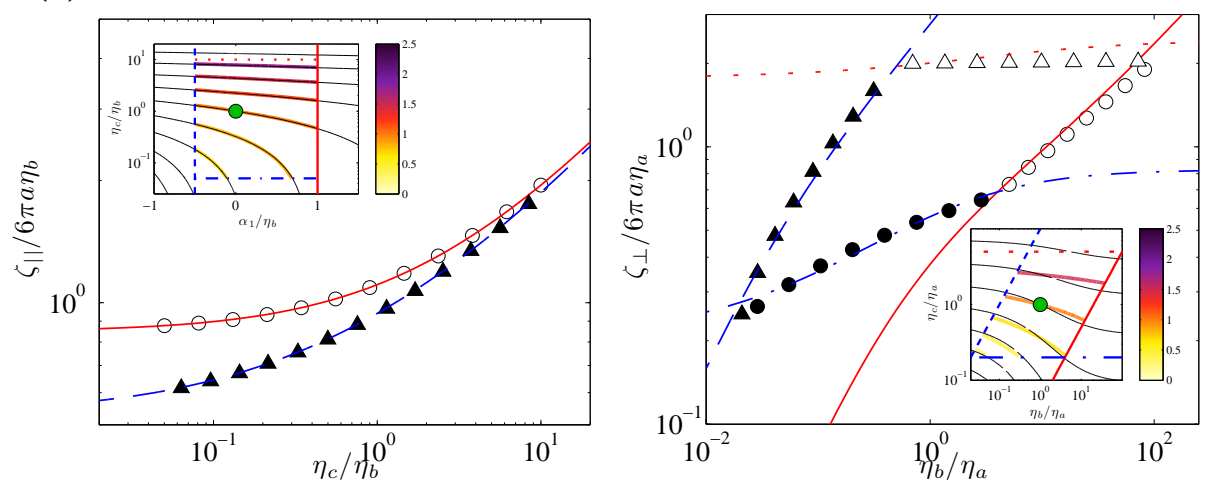

Figure 5: Comparison between our analytical expression for the response function (lines) and numerical simulations by (Heuer et al., 1992) (symbols). The two line plots on each figure represent the lower and upper limits of the parameter ranges provided in (Heuer et al., 1992). These limits and the entire parameter ranges are represented in the inset contour maps, where the thick colour contours come from the simulations, the thin black contours come from equations (23) and (24), and the green circle denotes the isotropic case. (a) Response function in the nematic direction, $\zeta_{\|}$, normalized with $a \eta_{b}$ and represented as a function of $\eta_{c} / \eta_{b}$ and $\alpha_{1} / \eta_{b}$ : - - and $\boldsymbol{\Lambda}, \alpha_{1}=-0.5 \eta_{b}$; - and $\circ, \alpha_{1}=\eta_{b} ;-.-, \eta_{c}=0.05 \eta_{b} ; \cdots \cdots, \cdot, \eta_{c}=10 \eta_{b}$. (b) Response function perpendicular to the nematic direction, $\zeta_{\perp}$, computed for $\alpha_{1}=0$, normalized with $a \eta_{a}$ and represented as a function of $\eta_{b} / \eta_{a}$ and $\eta_{c} / \eta_{a}:---$ and $\mathbf{\Delta}, \eta_{c}=10 \eta_{b}$; - and $\circ, \eta_{b}=0.05 \eta_{c} ;-$ - and $\bullet, \eta_{c}=0.2 \eta_{a} ; \cdots \cdots$ and $\triangle, \eta_{c}=5 \eta_{a}$.

creeping flow of a nematic liquid of uniform director around a sphere using finite differences and a relaxation time integrator. They provide empirical formulae for both $\zeta_{\|}$and $\zeta_{\perp}$ that fit their simulation results near the isotropy point.

Figure 5(a) compares the analytical expression for $\zeta_{\|} 23$ with Heuer et al.'s empirical fit in the parameter range where these authors find the fit to be accurate, that is $0.05<\eta_{c} / \eta_{b}<10$ and $-1 / 2<\alpha_{1} / \eta_{b}<1$ (Heuer et al., 1992). Both results are in an excellent agreement, not only around the isotropy point but in the whole domain, including its boundaries $\alpha_{1}=-\eta_{b} / 2$ and $\alpha_{1}=\eta_{b}$.

Figure $5(b)$ displays a similar comparison for $\zeta_{\perp}$, this time in the domain $0.2<\eta_{c} / \eta_{b}<5,0.2<\eta_{c} / \eta_{a}<5$ and $\alpha_{1}=0$, which is the range of accuracy of Heuer et al.'s empirical fit to their simulation data (Heuer et al., 1992). The agreement is again perfect near the isotropy point and for low values of $\eta_{b} / \eta_{a}$ and $\eta_{c} / \eta_{a}$ (bottom left corner of figure $5(b)$ ). However, it worsens slightly for high values of $\eta_{b} / \eta_{a}$ and $\eta_{c} / \eta_{a}$ (top right corner of the figure), where the simulation results seem to underestimate $\zeta_{\perp}$. These differences are however small and 
remain below $12 \%$. The observed divergence may be due to imprecisions in Heuer et al.'s fit to their own simulation results far from the isotropy point. This hypothesis is supported by comparing their simulation results to the prediction from the empirical fit for the one case in their paper where both are available, i.e. $\left(\eta_{b} / \eta_{a}, \eta_{c} / \eta_{a}, \alpha_{1} / \eta_{a}\right)=(1 / 3,10 / 3,0)$. For this combination of viscosities, the simulation yields $\zeta_{\perp} / 6 \pi a \eta_{a}=5.28$, which is in excellent agreement with our calculation of $\zeta_{\perp} / 6 \pi a \eta_{a}=5.27$. However, the empirical fit predicts $\zeta_{\perp} / 6 \pi a \eta_{c}=$ 5.47 , which differs by $4 \%$ from the simulation result.

\section{Assessing Particle Tracking Microrheology of Nematic Bio-Polymer Networks}

This section employs the expressions for the response function derived in $\$ 4.1$ to evaluate the accuracy of existing PTM formulae when applied to pseudoisotropic nematic fluids. This analysis allows us to separately determine the ability of those formulae to estimate strain directional viscosities or bending viscosities.

PTM determines the viscosity of a fluid from the measured response function of embedded microparticles. Whether the motion of the particles is spontaneously driven by the thermal excitation of the fluid (passive PTM) or externally forced (active PTM), the fluid viscosity is estimated by fitting the measured response function to a model for the drag of a canonical particle. The simplest possible model is the Stokes's formula $\zeta=6 \pi a \eta$, which is valid for isotropic fluids (Mason and Weitz, 1995; Mason et al., 1997; Mason, 2000). A second, more recent approach that is applied to nematic biogels and liquid crystals consists of applying Stokes's equation separately in the two principal directions of the motion to obtain two effective viscosity coefficients (Stark and Ventzki, 2001, Loudet et al. 2004 Hasnain and Donald, 2006, Rogers et al., 2008; del Alamo et al. 2008, He et al. 2008),

$$
\zeta_{\|, \perp}=6 \pi a \eta_{\|, \perp}^{\mathrm{eff}} .
$$

From the statistical mechanics perspective, this approach has been justified on the grounds that the motion of the particle in one principal direction is uncorrelated from its motion in the second principal direction (del Álamo et al. 2008). From the point of view of fluid mechanics, the effective viscosities were proposed as friction coefficients that quantify overall directional diffusivity in a nematic fluid (Stark and Ventzki, 2001). However, their relation to the actual directional viscosities of the fluid has not been characterized yet. Figure 6 sheds light into this question with the aid of experimental data coming from reconstituted biopolymer gels (Hasnain and Donald, 2006; He et al., 2008) and live cells (del Álamo et al., 2008). The figure displays the ratio of viscosity coefficients in pseudo-isotropic fluids as a function of the ratio $\zeta_{\perp} / \zeta_{\|}$calculated in this study, together with the predictions from the isotropic Stokes's law and the effective viscosity approach (38). The range of values of $\zeta_{\perp} / \zeta_{\|}$measured in 

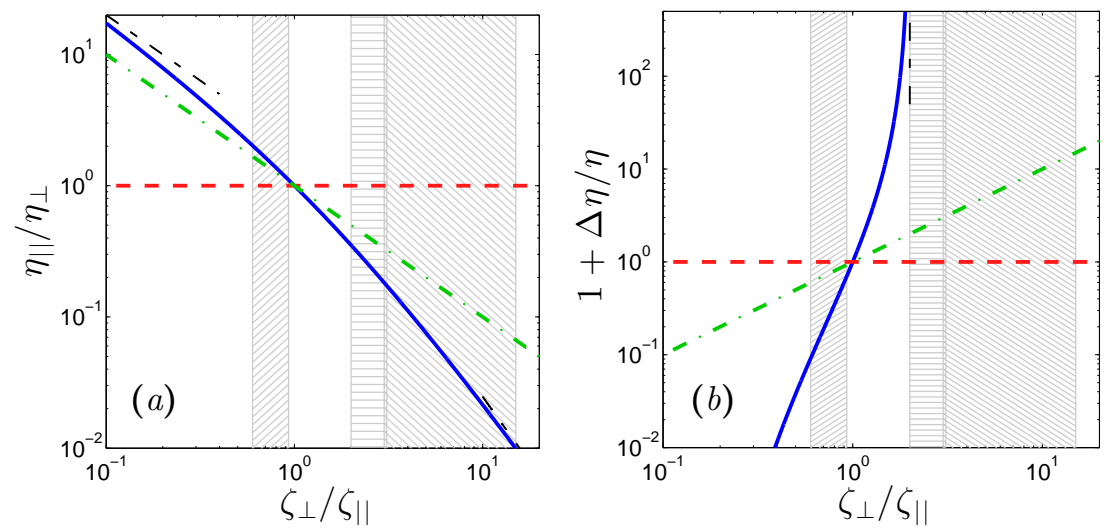

Figure 6: (a) Ratio of viscosity coefficients, $\eta_{\|} / \eta_{\perp}$, as a function of the ratio of directional response functions, $\zeta_{\perp} / \zeta_{\|}$. Each curve is obtained by using a different model for the response function: $--\_$, isotropic Stokes's formula; - - effective viscosity approach (equation 38); — fluid (see $\$ 4.1 .1) ;---$, asymptotes. The hatched areas represent experimental ranges of $\zeta_{\perp} / \zeta_{\|}$for biopolymer networks: left hatched, sheared DNA solutions (Hasnain and Donald, 2006); horizontally hatched, nematic reconstituted actin networks (He et al., 2008); right hatched, cytoplasm of live bovine aortic endothelial cells (del Alamo et al., 2008). (b) Same as panel (a) for a strain pseudo-isotropic fluid (see $\$ 4.1 .2$ ) where the relevant ratio of viscosities is $\Delta \eta / \eta$

experiments is included in the plots. In passive PTM experiments, this ratio is equal to the ratio of mean squared displacements of the particles $\left\langle\Delta x^{2}\right\rangle_{\perp} /\left\langle\Delta x^{2}\right\rangle_{\|}$ (del Álamo et al. 2008).

A first interesting observation that can be made from comparing figures 6( $a$ ) and $6(b)$ is that biopolymer networks, both reconstituted and inside live cells, behave closer to a rotationally pseudo-isotropic fluid than to a strain pseudoisotropic one. In fact, figure 6(b) reveals that most of the reported values of $\zeta_{\perp} / \zeta_{\|}$for these materials are disallowed by the strain pseudo-isotropic model. In this model, $\zeta_{\perp} / \zeta_{\|}$has a vertical asymptote at 2 and, thus, fluids where $\zeta_{\perp} / \zeta_{\|}$ is measured to be $>2$ are necessarily strain anisotropic. The only exception is the sheared DNA solution (Hasnain and Donald, 2006), which shows a nearly isotropic behavior that can be in principle consistent with both the rotationally pseudo-isotropic and the strain pseudo-isotropic conditions.

If, in consonance with the previous observation, one assumes that the rheology of nematic biopolymer networks is relatively close to rotationally pseudoisotropic, figure 6 (a) can be used to estimate the accuracy of previous response function models when applied to those biomaterials. Note that this estimation is not expected to be very precise as we merely proved that these fluids are not strain pseudo-isotropic, which is not exactly equivalent to proving that they are rotationally pseudo-isotropic. Nevertheless, we proceed with this estimation for 
lack of a better measure. Comparing the different curves in figure 6(a) within the range of experimental values of $\zeta_{\perp} / \zeta_{\|}$obtained from the literature indicates that previous models render errors of up to one order (effective viscosity) or two orders of magnitude (isotropic Stokes). In both cases, the actual anisotropy of the fluid, given by the ratio $\eta_{\perp} / \eta_{\|}$, is underestimated, with the effective viscosity approach predicting $0.8 \lesssim \eta_{\perp} / \eta_{\|} \lesssim 9$, and the present results predicting $0.5 \lesssim \eta_{\perp} / \eta_{\|} \lesssim 100$. Thus, researchers should exercise caution when interpreting directional PTM data.

\section{Influence of Anisotropy on the Far Velocity Field}

Anisotropy does not alter the $r^{-1}$ decay of the velocity far away from the sphere but it does modify the dependence of $\vec{v}$ on the azimuth and inclination angles. The velocity in the far field of the sphere can be calculated from equations (13) and $(22)$, yielding

$$
\vec{v}(\vec{x})=\frac{\overline{\overline{\mathcal{G}(\vec{x})}}}{8 \pi} \cdot\left[\begin{array}{ccc}
\zeta_{\|} & 0 & 0 \\
0 & \zeta_{\perp} & 0 \\
0 & 0 & \zeta_{\perp}
\end{array}\right] \cdot \vec{v}_{0}
$$

where $\vec{v}_{0}$ is the velocity of the particle. Similar to 4 , the Green's function is obtained by Fourier transforming equations (15)-(17) after regularizing with the Gaussian (21). This section focuses on the two basic flow configurations in which $\vec{v}_{0}$ is parallel and perpendicular to $\vec{n}$. All other possible configurations are linear combinations of these two basic flows.

\subsection{Velocity field caused by a sphere moving parallel to the nematic director $\left(\vec{v}_{0} \| \vec{n}\right)$}

Figure 7 displays velocity profiles around a sphere that translates parallel to the nematic director (i.e. northwards) and experiences a resistance equal to $\zeta_{\|}$. The velocity components parallel and perpendicular to the director, $v_{\|}$and $v_{\perp}$ respectively, are shown. These components fully define the axially symmetric velocity field elicited by the sphere. For illustrative purposes, we have represented $v_{\|}$and $v_{\perp}$ at a distance $r=5 a$ from its centre but similar results are obtained at other radial locations.

As noted above, the flow generated by $\vec{v}_{0} \| \vec{n}$ is independent of the first Miesowicz viscosity, so we restrict our analysis to the effect of the other two Miesowicz coefficients. For simplicity, we focus on the case $\alpha_{1}=0$ in which the equations governing the flow (29)-(30) become

$$
\begin{aligned}
& \partial_{x} p^{\prime}=\eta_{b} \nabla^{2} u_{x}, \\
& \partial_{r} p^{\prime}=\eta_{c}\left(\nabla^{2} u_{r}-u_{r} / r^{2}\right) .
\end{aligned}
$$


(a)

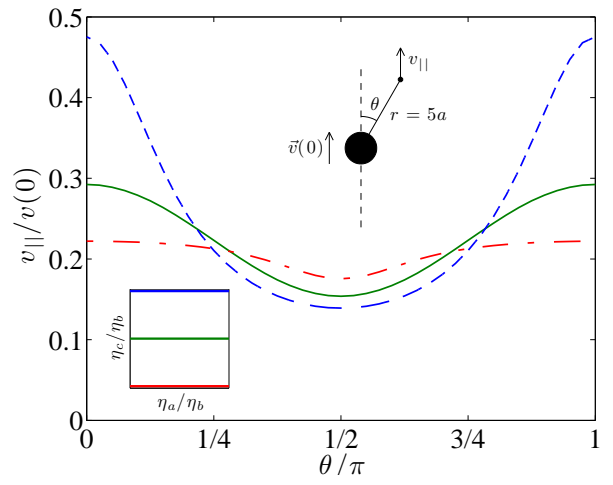

$(b)$

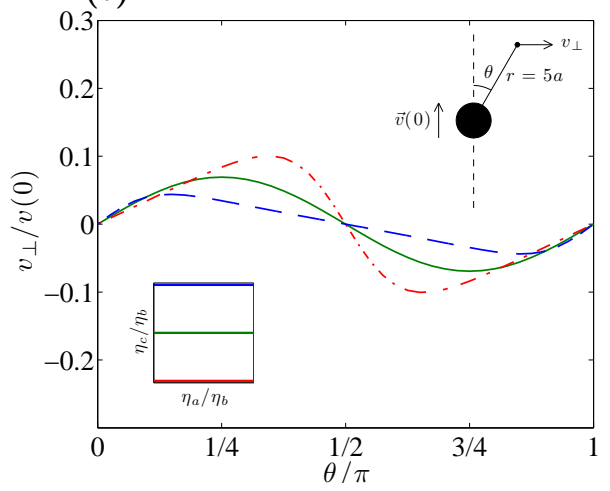

Figure 7: Longitudinal $\left(v_{\|}\right.$, panel $\left.a\right)$ and transverse $\left(v_{\perp}\right.$, panel $\left.b\right)$ flow velocity components elicited by a sphere moving parallel to the nematic director. The velocities are calculated at a distance $r=5 a$ away from the sphere centre, represented as a function of the inclination angle $\theta$. Each line type corresponds to a different value of $\eta_{c} / \eta_{b}$, as indicated in the inset map at the lower left corner of each figure:,$--- \eta_{c}=10 \eta_{b} ;-, \eta_{c}=\eta_{b} ;-\cdots, \eta_{c}=\eta_{b} / 10$.

These equations are similar to Stokes's equations but have different viscosity coefficients in the axial and radial directions. The resulting flow is therefore rotationally pseudo-isotropic, and $\eta_{b}$ and $\eta_{c}$ represent the viscosities in the $\|$ and $\perp$ directions, respectively (see equation 32 ). Curiously enough, invoking axial symmetry allows us to recast the very same equations as

$$
\begin{aligned}
& \partial_{x} p^{\prime}=\eta_{b} \nabla^{2} u_{x}, \\
& \partial_{r} p^{\prime}=\eta_{b}\left(\nabla^{2} u_{r}-u_{r} / r^{2}\right)+\left(\eta_{c}-\eta_{b}\right) \partial_{x} \omega_{\theta},
\end{aligned}
$$

which are now consistent with strain pseudo-isotropic conditions with $\eta_{b}=\eta$ and $\eta_{c}=\eta+\Delta \eta$ (see equation 35). The equivalence of these two formulations implies that the flow generated by a sphere moving parallel to the director is both strain pseudo-isotropic and rotationally isotropic. However, it is important to note that this conjunction arises exclusively from the flow geometry and not from the fluid properties, and that it does not mean that the flow is isotropic. In this configuration, the bending stresses are still different than zero and momentum diffuses differently in the $\|$ and $\perp$ directions unless $\eta_{c}=\eta_{a}$ and $\eta_{b}=\eta_{a}$, respectively. This is possible because the axial symmetry of the flow imposes a connection between the two types of anisotropic stresses, which is reflected by the equivalence $\eta_{\|}=\eta$ and $\eta_{\perp}=\eta+\Delta \eta$ that follows from comparing equations (39)-40 with equations 41)- (42). Consistent with this idea, the change of variables $\left(\eta_{\|}, \eta_{\perp}\right)=(\eta, \eta+\Delta \eta)$ transforms the rotationally pseudo-isotropic $\zeta_{\|}$ (33) into the strain pseudo-isotropic one (36). Of course, the same does not happen for $\zeta_{\perp}$ (equations 34 and 37 ) because the flow elicited by $\vec{v}_{0} \perp \vec{n}$ is not 


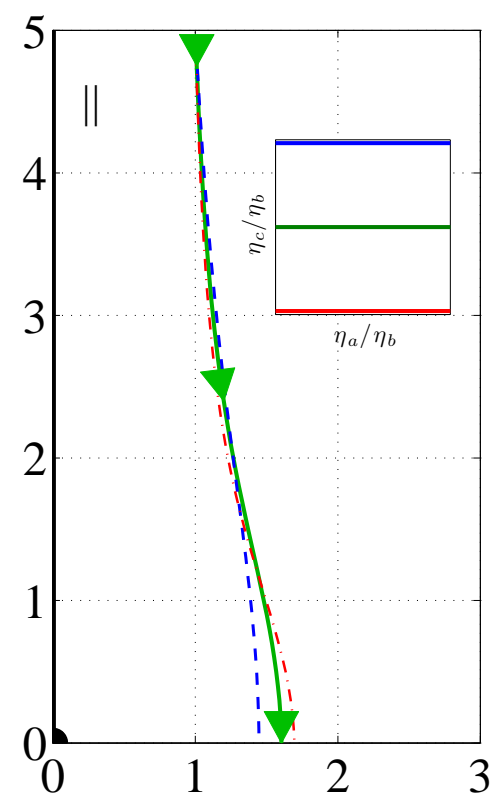

Figure 8: Streamlines on the meridional plane of a sphere that translates parallel to the nematic director (thick black vertical axis). The arrows indicate the flow direction in a reference frame moving with the sphere. The spatial coordinates are normalized with the sphere radius. One streamline is plotted for each value of $\eta_{c} / \eta_{b}$ considered in figure 7 - - - , $\eta_{c}=10 \eta_{b} ;-, \eta_{c}=\eta_{b}$; ,$-- \eta_{c}=\eta_{b} / 10$.

axially symmetric.

Probably due to this enhanced pseudo-isotropy, the velocity profiles in the $\vec{v}_{0} \| \vec{n}$ configuration conserve many isotropic features and the streamlines are not substantially affected by the viscosity ratio $\eta_{c} / \eta_{b}$. In particular, figure $7(a)$ shows that, regardless of the value of $\eta_{c} / \eta_{b}$, the longitudinal velocity component has a single maximum on the polar axis $(\theta=0)$ and a single minimum on the equatorial plane $(\theta=\pi / 2)$. The viscosity ratio $\eta_{c} / \eta_{b}$, however, has a marked effect on the peak-to-valley variation of $v_{\|}$. For $\eta_{c} / \eta_{b} \ll 1$, the spatial distribution of $v_{\|}$is nearly uniform. However, for $\eta_{c} \gg \eta_{b}$, there is a significant enhancement of the flow velocity on the polar axis, which is accompanied by a slight velocity damping on the equatorial plane.

The transverse velocity component is shown in figure $7(b)$. Owing to the symmetry of this flow configuration, $v_{\perp}$ is zero both on the polar axis and the equatorial plane. We find that the ratio $\eta_{c} / \eta_{b}$ influences both the maximum value of $v_{\perp}$ and its azimuthal location, which is $\theta=\pi / 4$ in the isotropic case. For $\eta_{c}<\eta_{b}$ the peak value of $v_{\perp}$ increases and its location is displaced towards the equator, whereas the opposite happens for $\eta_{c}>\eta_{b}$.

Figure 8 displays the flow streamlines obtained by integrating the far velocity 
field after subtracting the speed of the sphere. In order to illustrate the effect of anisotropy, the integration is performed for different values of $\eta_{c} / \eta_{b}$ from a common initial point far upstream of the sphere. It is found that anisotropy only has a modest influence on the flow streamlines when the sphere moves parallel to the nematic. Consistent with $\eta_{b}$ and $\eta_{c}$ acting as $\eta_{\|}$and $\eta_{\perp}$, respectively, in equations (39)-(40) and with the observed decrease of $v_{\perp}$ with $\eta_{c} / \eta_{b}$ (figure $7 b$ ), fluid particles approaching the sphere are deflected somewhat more in the $\perp$ direction for $\eta_{c}<\eta_{b}$ than in isotropic conditions, and the opposite happens for $\eta_{c}>\eta_{b}$. However, the level of deflection in this axially symmetric configuration is considerably smaller than when the sphere is moving perpendicular to the nematic (figure 11).

\subsection{Velocity field caused by a sphere moving perpendicu- lar to the nematic director $\left(\vec{v}_{0} \perp \vec{n}\right)$}

The flow generated by a sphere moving perpendicular to the nematic director (e.g.eastwards) is more complex than its $\vec{v}_{0} \| \vec{n}$ counterpart because axial symmetry is broken, the velocity field is three-dimensional and all four viscosity coefficients affect the flow. In this section, we study this dependence by plotting velocity profiles and streamlines for all of the possible combinations of the viscosity ratios defined by $\eta_{b} / \eta_{a}$ and $\eta_{c} / \eta_{a} \ll,=, \gg 1(0.1,1,10)$, and consistent with the scaling of the response function chosen to plot figure 3 . For simplicity, we restrict our analysis to $\alpha_{1}=0$ similar to the $\vec{v}_{0} \| \vec{n}$ configuration.

Figures 9 and 10 display angular profiles of the velocity at a constant distance $r=5 a$ from the origin, similar to figure 7. To characterise the spatial organization of the velocity field in the present three-dimensional configuration, we plot data on two planes. The first is the meridional plane $\phi=\pi / 2$ (figure 9) and the second is the equatorial plane $\theta=\pi / 2$ (figure 10). These planes intersect along the west-east axis of translation of the sphere. On them, $v_{\|}$is the velocity component parallel to the nematic director (i.e. northward), whereas $v_{\perp, \|}$ is perpendicular to the director and parallel to the sphere's trajectory (i.e. eastward). The remaining velocity component, $v_{\perp, \perp}$, is perpendicular to both the director and the sphere's trajectory.

Figure 11 demonstrates the effect of anisotropy on the streamline pattern. The streamlines in each panel are obtained by integrating the far velocity field relative to the sphere from a common initial point in the far east, upstream of the sphere. They are represented on the same meridional and equatorial planes used in figure 9 and 10 . Apart from allowing direct comparison between velocity profiles and streamlines, the choice of these two planes has the additional advantage that the local cross-plane flow velocity is zero, so that the streamlines remain in-plane. The same is not true for other initial points of integration given the three-dimensional nature of the flow when $\vec{v}_{0} \perp \vec{n}$. 
(a)

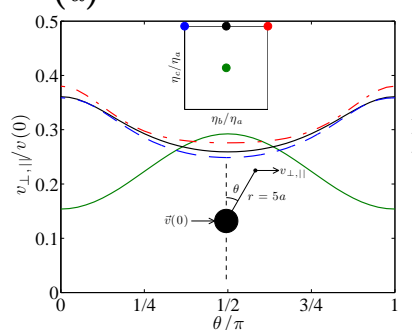

$(d)$

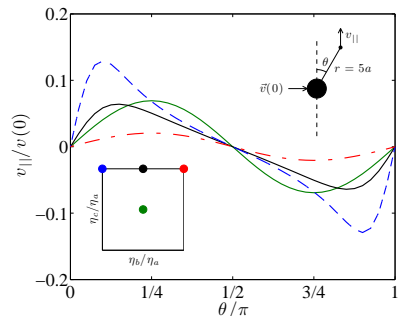

(b)

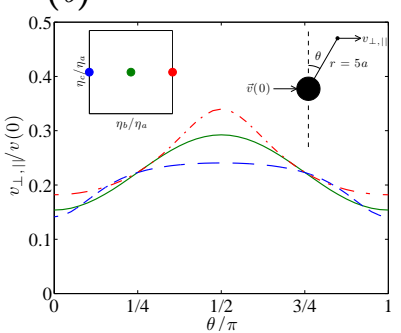

$(e)$

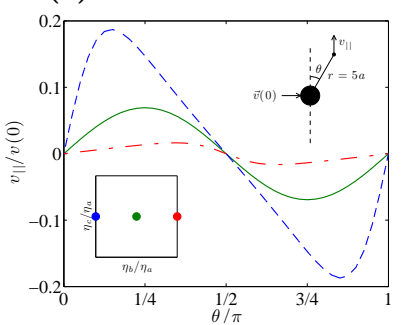

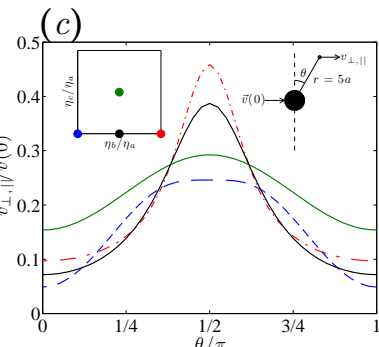

$(f)$

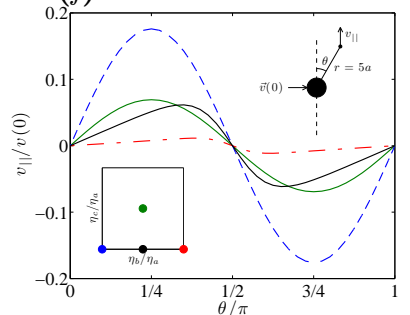

Figure 9: Flow velocity elicited by a sphere moving perpendicular to the nematic director. The velocities are calculated on the meridional plane $\phi=0$ at a distance $r=5 a$ away from the sphere centre, and represented as a function of the inclination angle $\theta:(a)-(c), v_{\perp, \|} ;(d)-(f), v_{\|} \cdot(a)$ and $(d), \eta_{a}=\eta_{c} / 10$; $(b)$ and $(e), \eta_{a}=\eta_{c} ;(f)$ and $(g), \eta_{a}=10 \eta_{c}$. Each line type corresponds to a different value of $\eta_{b} / \eta_{c}$, as indicated in the inset maps: ---, $\eta_{b}=\eta_{c} / 10$; ,$- \eta_{b}=\eta_{c} ;-.-, \eta_{b}=10 \eta_{c}$. The isotropic case is also plotted for reference $(-)$.
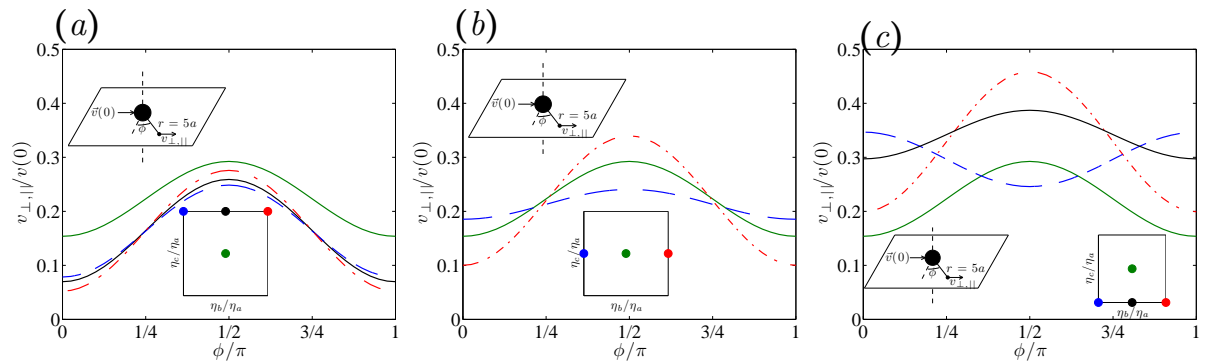

$(d)$
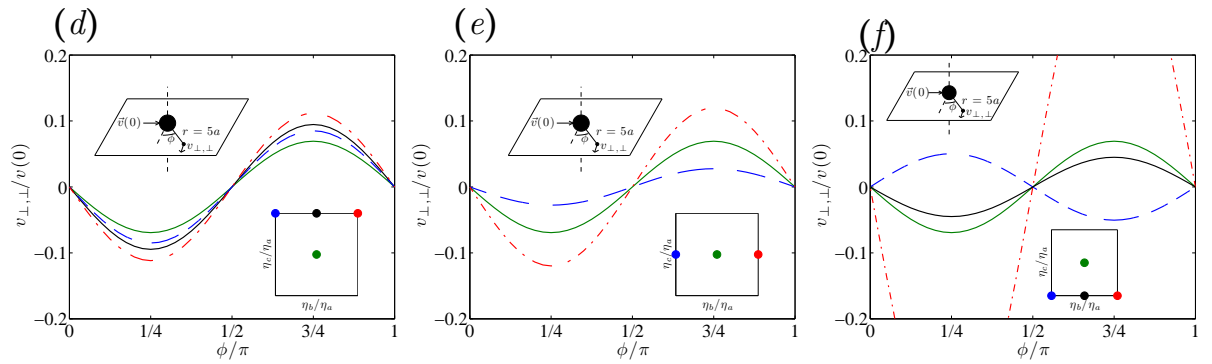

Figure 10: Same as Fig. 9 for the 2equatorial plane $\theta=0$. Velocities are represented as a function of the azimuth angle $\phi:(a)-(c), v_{\perp, \|} ;(d)-(f), v_{\perp, \perp}$. 


\subsubsection{Case $\eta_{a}=\eta_{c}$}

A first look into figures 9 and 10 indicates that, for $\eta_{a}=\eta_{c}$, the velocity profiles are qualitatively similar to the isotropic condition (panels $b$ and $e$ ). The reason for this behavior is that the bending stresses in the equations of fluid motion are zero for $\eta_{a}=\eta_{c}$, and the flow becomes rotationally pseudo-isotropic with $\eta_{b}$ and $\eta_{c}$ acting respectively as $\eta_{\|}$and $\eta_{\perp}$ (see equation 32). Comparing figures 7 and 8 with panels $(b),(e)$ of figures 9 and 10 and figure $11(b)$ suggests that anisotropy influences the flow organization more deeply in the present rotationally pseudoisotropic configuration than in the $\vec{v}_{0} \| \vec{n}$ case, consistent with the fact that the bending stresses are not zero in that configuration.

The response function of a sphere in the case $\eta_{a}=\eta_{c}$ is characterized in 4.1 .1 above. Here we focus on the effect of the ratio $\eta_{b} / \eta_{a}$ on the flow organization. We find that $v_{\perp, \mid l}$ increases with $\eta_{b} / \eta_{a}$ on the axis of translation of the sphere, $\theta=\pi / 2$, whereas it decreases or varies little on the perpendicular axes (figures $9 b$ and $10 b)$. Thus, the profile of this velocity component becomes sharper as $\eta_{b} / \eta_{a}$ increases. More importantly, the magnitude of $v_{\|}$decreases with $\eta_{b} / \eta_{a}$ on the meridional plane (figure 9e), while the magnitude of $v_{\perp, \perp}$ increases on the equatorial plane (figure $10 \mathrm{e}$ ). Overall, these velocity profiles suggest that the motion of the sphere leads to an asymmetric deflection of the fluid particles that is larger in the plane of lower viscosity and vice versa. This behavior can be easily appreciated by looking at the streamline pattern in figure 11(b). Note also that these streamlines resemble the streamlines in the flow of isotropic fluid around a prolate ellipsoid, consistent with the results in \$4.1.1

\subsubsection{Case $\eta_{c} \gg \eta_{a}$}

For large values of $\eta_{c} / \eta_{a}$, the viscous bending stresses become important in the equations of motion (6)-(8), causing changes in the anisotropic velocity field that depend little on the other viscosity ratio, $\eta_{b} / \eta_{a}$. This result is consistent with the fact that $\zeta_{\perp} /\left(a \eta_{a}\right)$ is almost independent of $\eta_{b} / \eta_{a}$ in the limit $\eta_{c} \gg \eta_{a}$ (see fig. 3 3 ). The largest variations in the velocity profiles occur on the meridional plane, where the east-west velocity component $v_{\perp, \|}$ increases substantially near the polar axis (figure 9a). This is also the region where the anisotropic stresses are maximum (data not shown). The northward velocity $v_{\|}$behaves qualitatively similar to the $\eta_{c}=\eta_{a}$ case. It experiences a moderate increase in magnitude and shifts towards the polar axis as $\eta_{b} / \eta_{a}$ decreases (figure $9 d$ ). As a consequence, the streamlines on the meridional plane are deflected more as $\eta_{b} / \eta_{a}$ decreases, and this deflection occurs closer to the location of the sphere (figure 11a).

The flow in the equatorial plane is even less sensitive to the ratio $\eta_{b} / \eta_{a}$, remaining very close to the isotropic case (figures 10a, $d$ ). In particular, the streamlines on this plane are almost identical to the isotropic ones, with a slight increase in deflection with $\eta_{b} / \eta_{a}$ (figure $11 a$ ). 
(a)

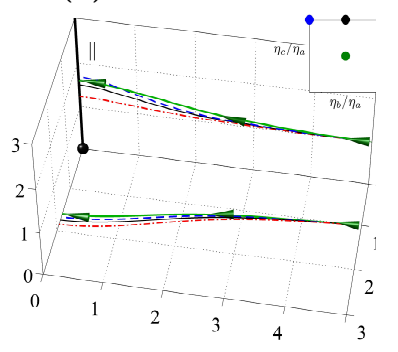

$(b)$

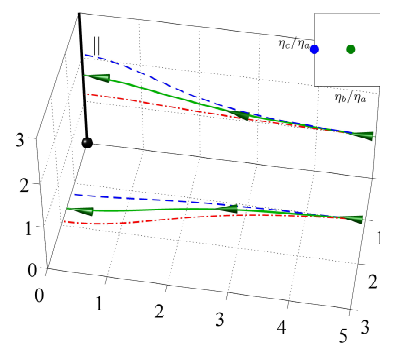

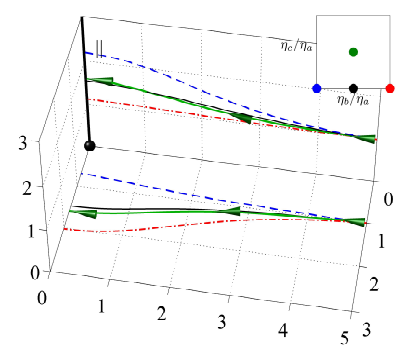

Figure 11: Streamlines on the meridional and equatorial planes of a sphere moving perpendicular to the nematic director (thick black vertical axis). The arrows indicate the flow direction in a reference frame moving with the sphere. The spatial coordinates are normalized with the sphere radius. Each panel corresponds to one value of the first viscosity ratio: $(a) \eta_{a}=\eta_{c} / 10 ;(b) \eta_{a}=\eta_{c}$; (c) $\eta_{a}=10 \eta_{c}$. Each line type corresponds to a different value of $\eta_{b} / \eta_{c}$, as indicated in the inset map at the upper right corner of each figure: - - , $\eta_{b}=\eta_{c} / 10 ;-, \eta_{b}=\eta_{c} ;-.-, \eta_{b}=10 \eta_{c}$. The isotropic case is also plotted for reference $(-)$.

\subsubsection{Case $\eta_{c} \ll \eta_{a}$}

Similar to the rotationally pseudo-isotropic condition, the flows with $\eta_{c} \ll \eta_{a}$ are rather sensitive to the ratio of momentum diffusivities in the $\|$ and $\perp$ directions, $\eta_{b} / \eta_{a}$. For large values of this ratio, $v_{\|}$decreases to virtually zero on the meridional plane (figure 9f), where the viscosity is higher $\left(\eta_{b}\right)$. Conversely, $v_{\perp, \perp}$ increases significantly on the equatorial plane (figure 10f), where the viscosity is lower $\left(\eta_{a}\right)$. Accordingly, the flow streamlines remain almost straight on the meridional plane whereas they are highly deflected away from the sphere on the equatorial plane (fig. 11c).

The velocity field corresponding to $\eta_{c} \ll \eta_{a}=\eta_{b}$ is comparable to the isotropic velocity field. The only remarkable difference is that the profile of $v_{\perp, \|}$ is sharper than the isotropic one on the meridional plane and flatter on the equatorial plane (figures $9 c$ and $10 c$ ). Apart from that, anisotropy affects little the velocity components perpendicular to axis of translation of the sphere in this case $\left(v_{\perp, \|}\right.$ and $v_{\|}$, figures $9 f$ and $\left.10 f\right)$, and the streamline pattern remains similar to the isotropic one (figure $11 c$ ).

Finally, we consider the flow elicited by a sphere moving perpendicular to the nematic director in a fluid with $\eta_{c} \ll \eta_{b} \ll \eta_{a}$. As expected, $v_{\|}$significantly increases in the meridional plane because of the $\eta_{b} \ll \eta_{a}$ condition (figure $9 f$ ), and the flow streamlines are highly deflected away from the sphere on this plane (figure 11 c). However, this flow has a remarkable characteristic which separates it from all other cases studied above, including the isotropic one; its 
streamlines converge towards the sphere on the equatorial plane (figure $11 c$ ). Consequently, the sign of $v_{\perp, \perp}$ on this plane is opposite to that of all of the other flow conditions (figure 10ff). This somewhat counterintuitive behavior can be partially understood by inspecting the balance of the different terms in the vorticity equation (9) at constant $\eta_{b} / \eta_{a}$ and varying $\eta_{c} / \eta_{a}$ (not shown). It is found that the angular profiles of the torques coming from the anisotropy of the stress-strain relation remain similar when varying $\eta_{c} / \eta_{a}$. However, the torques coming from the bending of the fluid with respect to the nematic change sign at $\eta_{c}=\eta_{a}$, attenuating the effect of the stress-strain anisotropy for $\eta_{c}>\eta_{a}$ and magnifying it for $\eta_{c}<\eta_{a}$.

\subsubsection{A remark on $\eta_{b} / \eta_{a}$ dependence of the flow}

It should be noted that the above classification in terms of $\eta_{c} / \eta_{a}$ is exclusively intended to facilitate the presentation of the nine flows obtained by combining $\eta_{c} / \eta_{a}=0.1,1,10$ and $\eta_{b} / \eta_{a}=0.1,1,10$ in the $\vec{v}_{0} \perp \vec{n}$ configuration. Likewise, one may choose to classify these flows in relation to the order of magnitude of $\eta_{b} / \eta_{a}$. Such classification is indeed implicit in figures 9 to 11 , where the strain pseudo-isotropic condition is depicted with solid (black) lines, whereas the $\eta_{b} / \eta_{a} \ll 1$ and $\eta_{b} / \eta_{a} \gg 1$ cases are depicted respectively by dashed (blue) and chain-dotted (red) lines. In broad strokes, although the general shape of the velocity profiles is mainly dependent on $\eta_{c} / \eta_{a}$, the velocity magnitudes in the direction perpendicular to the sphere's motion and, therefore, the asymmetry in streamline deflection, are mostly dependent on $\eta_{b} / \eta_{a}$.

\section{Conclusions}

\subsection{Summary of findings}

One of the greatest challenges that hinders the application of PTM to complex anisotropic fluids is the lack of knowledge about the dynamics of the probing particle. This paper studies the creeping flow generated by the motion of a point particle in a nematic incompressible fluid defined by the Leslie-Ericksen constitutive relation. The equations governing this flow are generally applicable to a broad range of nematic systems, ranging from liquid crystals to aligned biopolymer networks that are strongly coupled to their solvent, and nematic visco-elastomers.

The response function $\overline{\bar{\zeta}}$ of a sphere in a nematic fluid is a $3 \times 3$ tensor that provides the drag force $\vec{F}$ experienced by the particle as

$$
\vec{F}=\overline{\bar{\zeta}} \cdot \vec{v}_{0}
$$

where $\vec{v}_{0}$ is the velocity of the particle. This tensor only has two independent values, $\zeta_{\|}$and $\zeta_{\perp}$, which respectively represent the resistance of the fluid to the motion of the particle in the directions parallel and perpendicular to the nematic director, $\vec{n}$ (De Gennes and Prost, 1993). In this work, we provide 
$(a)$

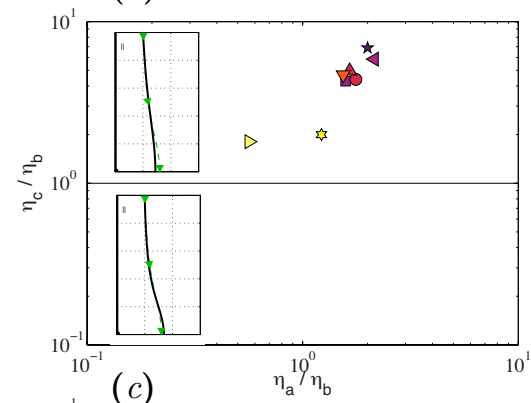

$(b)$

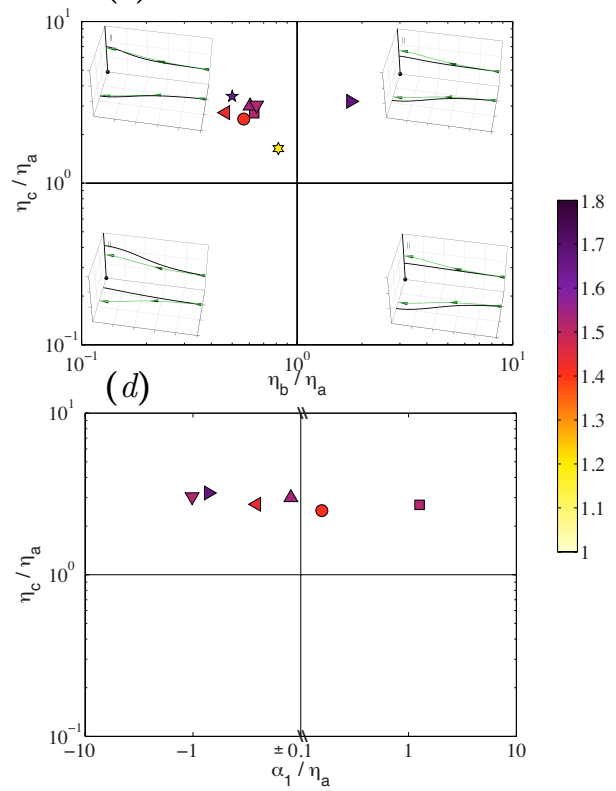

Figure 12: $\quad$ Survey of Miesowicz viscosity coefficients reported for nematic liquid crystals in the literature, plotted in the scaling of figures 2 and 3 ; $(a, c)$ $\zeta_{\|} / 6 \pi a \eta_{b} ;(b, d) \zeta_{\perp} / 6 \pi a \eta_{a}$. Each symbol represents a different compound and has been coloured according to the corresponding value of the response function as predicted by equations (23)-(24), and according to the colourbar on the right hand side of the figure: $\square$, PPA (Tseng et al., 1972, Orsay Liquid Crystal Group, 1971; Miesowicz, 1946); ○, MBBA (Gähwiller, 1971; Langevin, 1972); $\triangle$, 5CB (Skarp et al., 1980 Herba et al., 1985); $\nabla$, MBPP (Herba et al., 1985); $\triangleleft$, E7 (Wang et al., 2006); $\triangleright$, UCF-02 (Wang et al., 2006); $\star$, N4 (Beens and de Jeu, 1983); *, 8OCB (Janik et al., 1998). ( $a, b)$ also include the representative streamline pattern corresponding to each region of the parameter space. For reference, the isotropic streamlines are also included in green. 
explicit analytical expressions for $\zeta_{\|}$and $\zeta_{\perp}$, i.e. the equivalent of Stokes' drag formula for a nematic fluid, by performing a multipole expansion in Fourier space. These formulae depend on up to four viscosity coefficients: the three Miesowicz viscosities $\eta_{a}, \eta_{b}$ and $\eta_{c}$, and the first Leslie viscosity $\alpha_{1}$. Our solution compares well with the results from previous numerical simulations (Heuer et al. 1992 ) in the limited region of the parameter space where the simulation data are available. Similar to Kneppe et al. (1991), we observe that the flow caused by a particle moving parallel to the nematic $\left(\vec{v}_{0} \| \vec{n}\right)$ is axially symmetric and $\zeta_{\|}$is independent of the first Miesowicz viscosity. On the other hand, the flow originated by a particle moving perpendicular to the nematic $\left(\vec{v}_{0} \perp \vec{n}\right)$ is threedimensional and $\zeta_{\perp}$ depends on all four viscosity coefficients. It is important to note, however, that the $\vec{v}_{0} \| \vec{n}$ flow is independent of $\eta_{a}$ only if the velocities of the network and the solvent are divergence-free. This requirement is only satisfied when the viscous coupling between the network and the solvent is strong and the network velocity matches that of the incompressible solvent.

In a nematic two-fluid gel, anisotropy can present itself through the viscous coupling between the network and the solvent, as well as through the network's constitutive equations. The anisotropy arising from the coupling has been illustrated by considering an idealized network formed by a square array of circular cylinders, which opposes twice as much resistance to the relative motion of the solvent in the $\perp$ direction than in the $\|$ direction. Further analysis of the effect of anisotropic coupling will require relaxing the strong coupling assumption and is beyond the scope of this study. The anisotropy of the Leslie-Ericksen constitutive relation can, however, be thoroughly studied in the present formulation. We find that the response function is affected mainly by the anisotropic diffusion of momentum along different directions (i.e. anisotropy in the stressstrain relationship), and by the resistance of the fluid to bending. Analysis of the equations of fluid motion indicates that momentum diffusivity is anisotropic when $\eta_{a} \neq \eta_{b}$ and that the fluid opposes resistance to bending when $\eta_{a} \neq \eta_{c}$. We have studied these two mechanisms independently from each other by examining the response function in a rotationally pseudo-isotropic fluid where the bending stresses are zero and in a strain pseudo-isotropic fluid where the stress-strain relation is isotropic. The first of these fluids is governed by a pseudo-Stokes equation with different viscosities $\eta_{\|}=\eta_{b}$ and $\eta_{\perp}=\eta_{a}$ in the directions parallel and perpendicular to the nematic director. The second fluid is characterized by an isotropic momentum diffusivity $\eta=\eta_{a}$ plus a resistance to bending $\Delta \eta=\eta_{c}-\eta_{a}$. A comparative analysis of these two "pure" conditions indicates that anisotropic momentum diffusivity leads to greater changes in $\zeta_{\|}$, whereas $\zeta_{\perp}$ is more influenced by the bending resistance of the fluid.

It is also found that directional momentum diffusivity has a limited effect on the response function in rotationally pseudo-isotropic fluids, so that $\zeta_{\|} / \eta_{\|}$ and $\zeta_{\perp} / \eta_{\perp}$ reach constant asymptotic values for high levels of anisotropy with the only exception of $\zeta_{\|} / \eta_{\|}\left(\eta_{\|} \ll \eta_{\perp}\right)$. In fluids with zero resistance to bending, this result provides some level of justification for the qualitative usage of effective directional viscosities, which are defined by applying Stokes's drag law separately along the $\|$ and $\perp$ directions. However, it is important to note that 
the thus estimated effective viscosities differ from the actual viscosities of the fluid by over one order of magnitude for moderate levels of anisotropy like those observed in reconstituted biopolymer networks and the cytoskeleton of cultured animal cells. Furthermore, the very notion of directional effective viscosities may be misleading in fluids with an appreciable resistance to bending because the effect of $\Delta \eta$ is felt in both components of the response function for all levels of anisotropy.

The present study is finalized by a parametric description of the far velocity field around a spherical particle under different anisotropy conditions. For simplicity and due to the observed small influence of the first Leslie viscosity on the response function, the analysis is restricted to zero values of this coefficient $\left(\alpha_{1}=0\right)$. When the particle moves parallel to the nematic director, the axial symmetry of the flow establishes a constraint between the bending stresses in the fluid and the directional diffusion of momentum, which cannot vary independently of each other in this flow configuration. As a consequence, the flow pattern varies little with respect to the isotropic case although the level of streamline deflection increases when $\eta_{b}=\eta_{\|}>\eta_{\perp}=\eta_{c}$ and vice versa. When the sphere moves perpendicular to the nematic director, the flow is three-dimensional and the directional diffusion of momentum can be varied independently of the bending resistance of the fluid through the viscosity ratios $\eta_{b} / \eta_{a}$ and $\eta_{c} / \eta_{a}$. When $\eta_{c} / \eta_{a} \gg 1$, the fluid opposes high resistance to bending and the flow differs little from the isotropic case, similar to the $\vec{v}_{0} \| \vec{n}$ flow. However, when $\eta_{c} / \eta_{a}$ is of order unity, anisotropic momentum diffusivity can lead to large changes in the flow structure which essentially consist of asymmetric streamline deflection; for $\eta_{b}>\eta_{a}$, the streamlines are deflected more in the equatorial plane than in the meridional ones, and the opposite happens for $\eta_{b}<\eta_{a}$. Intriguingly, when $\eta_{c} / \eta_{a} \ll 1$, the resistance to bending enhances the flow asymmetry caused by anisotropic momentum diffusivity rather than reducing it, so much as to cause the streamlines to converge towards the sphere in the equatorial plane when $\eta_{b} \ll \eta_{a}$.

The parametric study of anisotropic viscosity effects on the flow of a nematic fluid around a sphere would be incomplete without a survey of the experimental values of the viscosity coefficients reported in the literature. Such a survey is useful in determining which of the conditions explored in this paper are more likely to be observed experimentally and, at the same time, it can offer new insight about the dynamics of those fluids. Unfortunately, there is little information about the anisotropic rheology of nematic biopolymer gels, and most of this information comes from effective viscosity approaches that suffer from the limitations described above. Our analysis suggests that these biopolymers are relatively close to a rotationally pseudo-isotropic fluid with $0.5 \lesssim \eta_{\perp} / \eta_{\|} \lesssim 100$ but these values should only be taken as rough estimations. For nematic liquid crystals, the situation is better and we find values of the Miesowicz viscosities for eight different compounds. This information is summarized in figure 12 using the same scaling as figures 2 and 3 . The vast majority of the compounds have $\eta_{c} / \eta_{a} \approx 3$ and $0.5 \lesssim \eta_{b} / \eta_{a} \lesssim 0.8$, which implies that their bending resistance is relatively high and that, in contrast to biopolymer networks, nematic 
liquid crystals can be considered to behave somewhat closer to a strain pseudoisotropic fluid than to a rotationally pseudo-isotropic fluid. Interestingly, all of the liquid crystals considered are in the range where $\alpha_{1}$ has a small effect on the flow.

Although the main motivation for this study is to provide fundamental understanding of the dynamics of a microrheology probe in nematic fluids, many of the results presented here have broader applications. Modelling the motility of spermatozoa in the cervical mucus is just one example of such applications. This mucus varies through different phases along the ovulatory cycle, including one in which the mucin network aligns parallel to the direction of the reproductory tract thereby favouring the directed migration of spermatozoa (Chretien, 2003). This anisotropic synergy could be studied in more detail by modeling each swimmer as a superposition of regularized point forces using the anisotropic Green's functions derived in this study.

\subsection{Model limitations}

The present formulation is linear, incompressible and assumes affine deformations. Thus, the results in this paper are valid only for small deformations and do not apply to regimes displaying strain-stiffening, negative normal stresses and other non-linear effects.

A potential limitation of this study is that, for the sake of brevity, we consider the response of the fluid to be purely viscous. However, most biopolymer gels have viscoelastic properties defined by a dependence of their stress field on their deformation at previous instants of time. A standard procedure to model these effects is to replace the constant viscosity coefficients in equation (5) by time-dependent memory functions, and the products by temporal convolutions. Owing to the linear nature of the problem, Fourier transforming in time renders similar equations in which the constant, real-valued viscosity coefficients are replaced by complex-valued functions of the frequency, $\alpha_{i} \rightarrow \widehat{\alpha}_{i}(s)$. From there, the calculation of the response function in the frequency domain follows the same steps as in the purely viscous case, although its analysis is complicated by the fact that both the viscosity coefficients and the response function are complex numbers. Such analysis is straightforward but is out of the scope of this paper.

One of the main potential limitations of this study is our assumption that the particle does not distort the nematic director field. Molecular anchoring at the particle surface may distort the director in liquid crystals, leading to dipole or Saturn ring configurations next to the particle. The drag force of a sphere in these configurations has been numerically calculated by Ruhwandl and Terentjev (1996), and Stark and Ventzki (2001), showing considerable differences with respect to results obtained for a uniform nematic. The influence of surface anchoring on the local director topology and particle diffusion has been determined experimentally (Koenig et al., 2009) and numerically (Moreno-Razo et al., 2011). These near-field effects disappear in two-point PTM (Crocker 
et al. 2000, Levine and Lubensky, 2001), which is based on measurements of correlated motion of distant particles.

Particle motion can also distort the director in the far field due to the finite elastance of the nematic. We have hypothesized that this effect is negligible in passive PTM of biopolymer networks because the thermal energy that drives particle motion is extremely low. However, this energy can be several orders of magnitude higher in active PTM. In that case, the methodology presented here can be extended by prescribing a suitable spatiotemporal nematic distribution (Stark and Ventzki, 2001), including the Frank elasticity constants into the formulation (Frank, 1958) or by calculating the dynamics of the nematic through the Poisson-bracket approach (Stark and Lubensky, 2003).

Finally, assuming a uniform director is relatively reasonable for live cell PTM experiments. Adherent cells are known to align their cytoskeletal fibers in response to directional mechanical stimuli (Galbraith et al., 1998; Kaunas et al. 2005) and subtratum stiffness (Lee et al. 2013). Even in the absence of global alignment, the cytoskeleton will organize in smaller nematic domains in which the director remains locally uniform.

\section{Acknowledgements}

This work was supported by NSF CAREER grant CBET-1055697. Manuel Gómez-González was partially funded by Fundación Ibercaja. The authors are indebted to Dr. Shu Chien, Dr. Juan C. Lasheras, Dr. Javier Jimenez and Dr. Javier Urzay for fruitful discussions on preliminary versions of this manuscript.

\section{A Green's Functions}

In this appendix, we outline the procedures to obtain the Green's functions for the flow velocity and pressure from the equations of motion $[10, \sqrt{11}, \sqrt{12}$ and (3). In Fourier space, these equations of motion become

$$
\begin{array}{rlrl}
i k_{1} \widehat{p}+\left(\frac{1}{2 \pi}\right)^{3 / 2} \widehat{f_{x}} & =\left(\eta_{c}+\eta_{b}-\eta_{a}+\alpha_{1}\right) k_{1}^{2} \widehat{u}+\eta_{b}\left(k_{2}^{2}+k_{3}^{2}\right) \widehat{u}, \\
i k_{2} \widehat{p}+\left(\frac{1}{2 \pi}\right)^{3 / 2} \widehat{f_{y}} & = & \eta_{c} k_{1}^{2} \widehat{v}+\eta_{a}\left(k_{2}^{2}+k_{3}^{2}\right) \widehat{v}, \\
i k_{3} \widehat{p}+\left(\frac{1}{2 \pi}\right)^{3 / 2} \widehat{f_{z}}= & \eta_{c} k_{1}^{2} \widehat{w}+\eta_{a}\left(k_{2}^{2}+k_{3}^{2}\right) \widehat{w}, \\
k_{1} \widehat{u}+k_{2} \widehat{v}+k_{3} \widehat{w} & =0 . &
\end{array}
$$

Assuming a linear solution of the form 13 - 14 , equations 43 - 46 
simplify to

$$
\begin{aligned}
{\left[\left(\alpha_{1}+\eta_{c}-\eta_{a}\right) k_{1}^{2}+\eta_{b} k^{2}\right] \frac{\widehat{\mathcal{G}}_{1 j}}{8 \pi} } & =i k_{1} \frac{\widehat{\mathcal{P}}_{j}}{8 \pi}+\left(\frac{1}{2 \pi}\right)^{3 / 2} \delta_{1 j}, \\
{\left[\left(\eta_{c}-\eta_{a}\right) k_{1}^{2}+\eta_{a} k^{2}\right] \frac{\widehat{\mathcal{G}}_{2 j}}{8 \pi} } & =i k_{2} \frac{\widehat{\mathcal{P}}_{j}}{8 \pi}+\left(\frac{1}{2 \pi}\right)^{3 / 2} \delta_{2 j}, \\
{\left[\left(\eta_{c}-\eta_{a}\right) k_{1}^{2}+\eta_{a} k^{2}\right] \frac{\widehat{\mathcal{G}}_{3 j}}{8 \pi} } & =i k_{3} \frac{\widehat{\mathcal{P}}_{j}}{8 \pi}+\left(\frac{1}{2 \pi}\right)^{3 / 2} \delta_{3 j}, \\
k_{i} \widehat{\mathcal{G}}_{i j} & =0 .
\end{aligned}
$$

After applying the divergence of equations (47) - 49) and using (50), we obtain

$$
\frac{\widehat{\mathcal{P}}_{j}}{8 \pi}=\left(\frac{1}{2 \pi}\right)^{3 / 2} \frac{i k_{j}}{k^{2}}-\left[\alpha_{1} k_{1}^{2}+\left(\eta_{b}-\eta_{a}\right) k^{2}\right] \frac{i k_{1}}{k^{2}} \frac{\widehat{\mathcal{G}_{1 j}}}{8 \pi} .
$$

Finally, plugging this result into equations 447 - 49) and (51) we obtain the Green's functions for the velocity and pressure,

$$
\begin{aligned}
& \frac{\widehat{\mathcal{G}}_{1 j}}{\sqrt{8 / \pi}}=\frac{\delta_{1 j} k^{2}-k_{1} k_{j}}{\alpha_{1} k_{1}^{2}\left(k_{2}^{2}+k_{3}^{2}\right)+\eta_{b} k^{4}+\left(\eta_{c}-\eta_{b}\right) k_{1}^{2} k^{2}}, \\
& \frac{\widehat{\mathcal{G}}_{2 j}}{\sqrt{8 / \pi}}=\frac{\delta_{2 j}}{\left(\eta_{c}-\eta_{a}\right) k_{1}^{2}+\eta_{a} k^{2}}-k_{2} k_{j} \frac{\left(1-\delta_{1 j}\right) \frac{\alpha_{1} k_{1}^{2}+\left(\eta_{b}-\eta_{a}\right) k^{2}}{\left.\eta_{c}-\eta_{a}\right) k_{1}^{2}+\eta_{a} k^{2}}+1}{\alpha_{1} k_{1}^{2}\left(k_{2}^{2}+k_{3}^{2}\right)+\eta_{b} k^{4}+\left(\eta_{c}-\eta_{b}\right) k_{1}^{2} k^{2}}, \\
& \frac{\widehat{\mathcal{G}}_{3 j}}{\sqrt{8 / \pi}}=\frac{\delta_{3 j}}{\left(\eta_{c}-\eta_{a}\right) k_{1}^{2}+\eta_{a} k^{2}}-k_{3} k_{j} \frac{\left(1-\delta_{1 j}\right) \frac{\alpha_{1} k_{1}^{2}+\left(\eta_{b}-\eta_{a}\right) k^{2}}{\left.\eta_{c}-\eta_{a}\right) k_{1}^{2}+\eta_{a} k^{2}}+1}{\alpha_{1} k_{1}^{2}\left(k_{2}^{2}+k_{3}^{2}\right)+\eta_{b} k^{4}+\left(\eta_{c}-\eta_{b}\right) k_{1}^{2} k^{2}}, \\
& \frac{\widehat{\mathcal{P}}_{j}}{\sqrt{8 / \pi}}=i k_{j}\left[\frac{1-\delta_{1 j}}{k^{2}}+\frac{\frac{1-\delta_{1 j}}{k^{2}}\left(\alpha_{1} k_{1}^{4}-\eta_{a} k^{4}\right)+\eta_{a} k^{2}+\left(\eta_{b}-\eta_{a}\right) k_{1}^{2}+\left(\eta_{c}-\eta_{b}\right) k_{1}^{2} \delta_{1 j}}{\alpha_{1} k_{1}^{2}\left(k_{2}^{2}+k_{3}^{2}\right)+\eta_{b} k^{4}+\left(\eta_{c}-\eta_{b}\right) k_{1}^{2} k^{2}}\right] .
\end{aligned}
$$

\section{B Expressions for the Functions Appearing in the Response Function}

Equations 223 and (24) for the response function of a sphere in a nematic fluid refer to the non-dimensional functions

$$
\begin{aligned}
& A(\vec{\eta})=\frac{\alpha_{1}}{\eta_{b}}+\frac{\eta_{c}}{\eta_{b}}-1, \\
& B(\vec{\eta})=\sqrt{A(\vec{\eta})^{2}+4 \frac{\alpha_{1}}{\eta_{b}}},
\end{aligned}
$$




$$
\begin{gathered}
C_{+}(\vec{\eta})=\sqrt{\frac{A(\vec{\eta})+B(\vec{\eta})}{2}}, \\
C_{-}(\vec{\eta})=\sqrt{\frac{A(\vec{\eta})-B(\vec{\eta})}{2}}, \\
D_{+}(\vec{\eta})=A(\vec{\eta})+2+B(\vec{\eta}), \\
D_{-}(\vec{\eta})=A(\vec{\eta})+2-B(\vec{\eta}), \\
E_{+}(\vec{\eta})=A(\vec{\eta}) D_{+}(\vec{\eta})-2\left(\frac{\eta_{c}}{\eta_{b}}-1\right), \\
E_{-}(\vec{\eta})=A(\vec{\eta}) D_{-}(\vec{\eta})-2\left(\frac{\eta_{c}}{\eta_{b}}-1\right),
\end{gathered}
$$

which arise from the integrals in equation $(20)$.

These equations degenerate in the limit $B(\vec{\eta}) \longrightarrow 0$, which occurs whenever

$$
\frac{\eta_{c}}{\eta_{b}} \longrightarrow 1-\frac{\alpha_{1}}{\eta_{b}} \pm 2 \sqrt{\frac{-\alpha_{1}}{\eta_{b}}} .
$$

In this limit, $C_{+} \rightarrow C_{-}, D_{+} \rightarrow D_{-}$and $E_{+} \rightarrow E_{-}$, and the expressions that determine the two components of the response function (equations $23 \mid 24$ become undefined. Solving the limit yields that $\zeta_{\|}=\zeta_{\perp}=0$ along the branch associated with the - sign in the square root of equation (56), and the same happens for the - branch when $\alpha_{1}>\eta_{b}$. As a consequence, the region of parameter space corresponding to

$$
\eta_{c}<\eta_{b}-\alpha_{1}-2 \sqrt{-\alpha_{1} \eta_{b}}, \quad \alpha_{1}<-\eta_{b}
$$

is associated with unphysical complex values of the response functions and cannot be realized. On the other hand, the signs of $\zeta_{\|}$and $\zeta_{\perp}$ do not change along the branch associated with the + sign in the square root of equation (56).

\section{References}

Beale, J. T. and Lai, M.-C. (2001). A method for computing nearly singular integrals. SIAM Journal on Numerical Analysis, 38(6):1902-1925.

Beens, W. W. and de Jeu, W. H. (1983). Flow-measurements of the viscosity coefficients of 2 nematic liquid-crystalline azoxybenzenes. Journal de physique, 44(2):129-136.

Brake, J. M., Daschner, M. K., Luk, Y.-Y., and Abbott, N. L. (2003). Biomolecular interactions at phospholipid-decorated surfaces of liquid crystals. Science, 302(5653):2094-2097. 
Chen, C. S., Tan, J., and Tien, J. (2004). Mechanotransduction at cell-matrix and cell-cell contacts. Annual Review of Biomedical Engineering, 6:275-302.

Chien, S. (2007). Mechanotransduction and endothelial cell homeostasis: the wisdom of the cell. American Journal of Physiology - Heart and Circulatory Physiology, 292(3):H1209-H1224.

Chretien, F. C. (2003). Involvement of the glycoproteic meshwork of cervical mucus in the mechanism of sperm orientation. Acta Obstetricia et Gynecologica Scandinavica, 82(5):449-461.

Cortez, R. (2001). The method of regularied stokeslets. SIAM Journal on Scientific Computing, 23(4):1204-1225.

Crocker, J. C. and Hoffman, B. D. (2007). Multiple-particle tracking and twopoint microrheology in cells. Methods in Cell Biology, 83:141-178.

Crocker, J. C., Valentine, M. T., Weeks, E. R., Gisler, T., Kaplan, P. D., Yodh, A. G., and Weitz, D. A. (2000). Two-point microrheology of inhomogeneous soft materials. Physical Review Letters, 85(4):888-891.

De Gennes, P. G. and Prost, J. (1993). The Physics of Liquid Crystals. Oxford University Press, second edition.

del Álamo, J. C., Norwich, G. N., Li, Y. J., Lasheras, J. C., and Chien, S. (2008). Anisotropic rheology and directional mechanotransduction in vascular endothelial cells. Proceedings of the National Academy of Sciences of the United States of America, 105(40):15411-15416.

Ericksen, J. L. (1960). Anisotropic fluids. Archive for Rational Mechanics and Analysis, 4(15):231-237.

Frank, F. C. (1958). On the theory of liquid crystals. Discussions of the Faraday Society, (25):19-28.

Fu, H. C., Shenoy, V. B., and Powers, T. R. (2008). Role of slip between a probe particle and a gel in microrheology. Physical Review E, 78(6):061503.

Gähwiller, C. (1971). The viscosity coefficients of a room-temperature liquid crystal (mbba). Physics Letters A, 36(4):311-312.

Galbraith, C. G., Skalak, R., and Chien, S. (1998). Shear stress induces spatial reorganization of the endothelial cell cytoskeleton. Cell Motility and the Cytoskeleton, 40(4):317-330.

Gittes, F., Schnurr, B., Olmsted, P. D., MacKintosh, F. C., and Schmidt, C. F. (1997). Microscopic viscoelasticity: Shear moduli of soft materials determined from thermal fluctuations. Physical Review Letters, 79(17):3286-3289.

Hasnain, I. A. and Donald, A. M. (2006). Microrheological characterization of anisotropic materials. Physical Review E, 73(3):031901. 
He, J., Mak, M., Liu, Y., and Tang, J. X. (2008). Counterion-dependent microrheological properties of f-actin solutions across the isotropic-nematic phase transition. Physical Review E, 78(1):011908.

Herba, H., Szymanski, A., and Drzymala, A. (1985). Experimental test of hydrodynamic theories for nematic liquid-crystals. Molecular Crystals and Liquid Crystals, 127(1):153-158.

Heuer, H., Kneppe, H., and Schneider, F. (1992). Flow of a nematic liquidcrystal around a sphere. Molecular Crystals and Liquid Crystals, 214:43-61.

Janik, J., Moscicki, J. K., Czuprynski, K., and Dabrowski, R. (1998). Miesowicz viscosities study of a two-component thermotropic mixture. Physical Review E, 58(3):3251-3258.

Kaunas, R., Nguyen, P., Usami, S., and Chien, S. (2005). Cooperative effects of Rho and mechanical stretch on stress fiber organization. Proceedings of the National Academy of Sciences of the United States of America, 102(44):1589515900 .

Kim, S. and Karrila, S. J. (2005). Microhydrodynamics - Principles and Selected Applications. Dover Publications, Inc.

Kneppe, H. and Schneider, F. (1981). Determination of the viscosity coefficients of the liquid-crystal mbba. Molecular Crystals and Liquid Crystals, 65(12):23-37.

Kneppe, H., Schneider, F., and Schwesinger, B. (1991). Axisymmetrical flow of a nematic liquid crystal around a sphere. Molecular Crystals and Liquid Crystals, 205:9-28.

Koenig, G. M., Ong, R., Cortes, A. D., Moreno-Razo, J. A., de Pablo, J. J., and Abbott, N. L. (2009). Single nanoparticle tracking reveals influence of chemical functionality of nanoparticles on local ordering of liquid crystals and nanoparticle diffusion coefficients. Nano Letters, 9(7):2794-2801.

Kole, T. P., Tseng, Y., Jiang, I., Katz, J. L., and Wirtz, D. (2005). Intracellular mechanics of migrating fibroblasts. Molecular Biology of the Cell, 16(1):328338.

Ladyzhenskaya, O. A. (1969). The Mathematical Theory of Viscous Incompressible Flow. Gordon and Breach Science Publishers, second english edition.

Lammerding, J. and Lee, R. T. (2005). The nuclear membrane and mechanotransduction: impaired nuclear mechanics and mechanotransduction in lamin a/c deficient cells. Novartis Found. Symp., 264:264-273.

Langevin, D. (1972). Spectrum analysis of light scattered from free-surface of a nematic liquid-crystal - surface-tension and viscosity measurements. Journal de physique, 33(2-3):249-256. 
Lee, H.-H., Lee, H.-C., Chou, C.-C., Hur, S. S., Osterday, K., del Álamo, J. C., Lasheras, J. C., and Chien, S. (2013). Shp2 plays a crucial role in cell structural orientation and force polarity in response to matrix rigidity. Proceedings of the National Academy of Sciences, 110(8):2840-2845.

Lee, J. S. H., Chang, M. I., Tseng, Y., and Wirtz, D. (2005). Cdc42 mediates nucleus movement and mtoc polarization in swiss 3 t3 fibroblasts under mechanical shear stress. Molecular Biology of the Cell, 16(2):871-880.

Leslie, F. M. (1966). Some constitutive equations for anisotropic fluids. Quarterly Journal of Mechanics and Applied Mathematics, 19(3):357-370.

Levine, A. J. and Lubensky, T. C. (2000). One- and two-particle microrheology. Physical Review Letters, 85(8):1774-1777.

Levine, A. J. and Lubensky, T. C. (2001). Response function of a sphere in a viscoelastic two-fluid medium. Physical Review E, 63(4):041510.

Lin, A., Krockmalnic, G., and Penman, S. (1990). Imaging Cytoskeleton Mitochondrial-membrane Attachments by Embedment-free Electronmicroscopy of Saponin-extracted Cells. Proceedings of the National Academy of Sciences of the United States of America, 87(21):8565-8569.

Liron, N. and Barta, E. (1992). Motion of a rigid particle in stokes flow: a new second-kind boundary-integral equation formulation. Journal of Fluid Mechanics, 238:579-598.

Loudet, J.-C., Barois, P., and Poulin, P. (2000). Colloidal ordering from phase separation in a liquid-crystalline continuous phase. Letters to Nature, 407(6804):611-613.

Loudet, J. C., Hanusse, P., and Poulin, P. (2004). Stokes drag on a sphere in a nematic liquid crystal. Science, 306(5701):1525-1525.

Luby-Phelps, K. (2000). Cytoarchitecture and physical properties of cytoplasm: Volume, viscosity. diffusion, intracellular surface area. In International Review of Cytology - A survey of Cell Biology, vol 192, volume 192 of International Review of Cytology - A survey of Cell Biology, pages 189-221. Academic Press Inc, San Diego, CA 92101-4495 USA.

Luby-Phelps, K., Taylor, D. L., and Lanni, F. (1986). Probing the Structure of Cytoplasm. Journal of Cell Biology, 102(6):2015-2022.

Mason, T. G. (2000). Estimating the viscoelastic moduli of complex fluids using the generalized stokes-einstein equation. Rheologica Acta, 39(4):371-378.

Mason, T. G., Ganesan, K., van Zanten, J. H., Wirtz, D., and Kuo, S. C. (1997). Particle tracking microrheology of complex fluids. Physical Review Letters, 79(17):3282-3285. 
Mason, T. G. and Weitz, D. A. (1995). Optical measurements of frequencydependent linear viscoelastic moduli of complex fluids. Physical Review Letters, 74(7):1250-1253.

McGrath, J. L., Hartwig, J. H., and Kuo, S. C. (2000). The mechanics of f-actin microenvironments depend on the chemistry of probing surfaces. Biophysical Journal, 79(6):3258-3266.

Miesowicz, M. (1936). Influence of the magnetic field on the viscosity of liquids in the nematic phase. Bulletin International de l'Academie Polonaise des Sciences et des Lettres, (5-6):228-247.

Miesowicz, M. (1946). The three coefficients of viscosity of anisotropic liquids. Nature, 158(4001):27-27.

Minin, A. A., Kulik, A. V., Gyoeva, F. K., Li, Y., Goshima, G., and Gelfand, V. I. (2006). Regulation of mitochondria distribution by rhoa and formins. Journal of Cell Science, 119(4):659-670.

Moreno-Razo, J. A., Sambriski, E. J., Koenig, G. M., Díaz-Herrera, E., Abbott, N. L., and de Pablo, J. J. (2011). Effects of anchoring strength on the diffusivity of nanoparticles in model liquid-crystalline fluids. Soft Matter, $7(15): 6828-6835$.

Orsay Liquid Crystal Group (1971). Viscosity measurements by quasi elastic light scattering in p-azoxyanisol. Molecular Crystals and Liquid Crystals, 13(2):187-191.

Parodi, O. (1970). Stress tensor for a nematic liquid crystal. Le Journal de Physique, 31(7):581-584.

Pokrovskii, V. N. and Tskhai, A. A. (1986). Slow motion of a particle in a weakly anisotropic viscous fluid. PMM Journal of Applied Mathematics and Mechanics, 50(3):391-394.

Pollard, T. D. and Borisy, G. G. (2003). Cellular motility driven by assembly and disassembly of actin filaments. Cell, 112(4):453-465.

Poulin, P., Stark, H., Lubensky, T. C., and Weitz, D. A. (1997). Novel colloidal interactions in anisotropic fluids. Science, 275(5307):1770-1773.

Rogers, S. S., Waigh, T. A., and Lu, J. R. (2008). Intracellular microrheology of motile amoeba proteus. Biophysical Journal, 94(8):3313-3322.

Ruhwandl, R. W. and Terentjev, E. M. (1996). Friction drag on a particle moving in a nematic liquid crystal. Physical Review E, 54(5):5204-5210.

Scherp, P. and Hasenstein, K. H. (2007). Anisotropic viscosity of the Chara (Characeae) rhizoid cytoplasm. American Journal Of Botany, 94(12):19301934. 
Skarp, K., Lagerwall, S. T., and Stebler, B. (1980). Measurements of hydrodynamic parameters for nematic 5cb. Molecular Crystals and Liquid Crystals, $60(3): 215-236$.

Squires, T. M. (2008). Nonlinear microrheology: bulk stresses versus direct interactions. Langmuir, 24(4):1147-1159.

Squires, T. M. and Mason, T. G. (2010). Fluid mechanics of microrheology. Annual Review of Fluid Mechanics, 42:413-438.

Stark, H. and Lubensky, T. C. (2003). Poisson-bracket approach to the dynamics of nematic liquid crystals. Physical Review E, 67(6):061709.

Stark, H. and Ventzki, D. (2001). Stokes drag of spherical particles in a nematic environment at low ericksen numbers. Physical Review E, 64(3):031711.

Stenull, O. and Lubensky, T. C. (2004). Dynamics of nematic elastomers. Physical Review E, 69(5):051801.

Su, J., Jiang, X., Welsch, R., Whitesides, G. M., and So, P. T. C. (2007). Geometric Confinement Influences Cellular Mechanical Properties I - Adhesion Area Dependence. Molecular \& Cellular Biomechanics, 4(2):87-104.

Tseng, H. C., Silver, D. L., and Finlayson, B. A. (1972). Application of the continuum theory to nematic liquid crystals. The Physics of fluids, 15(7):12131222 .

Tseng, Y., Kole, T. P., and Wirtz, D. (2002). Micromechanical mapping of live cells by multiple-particle-tracking microrheology. Biophysical Journal, 83(6):3162-3176.

Valentine, M. T., Perlman, Z. E., Gardel, M. L., Shin, J. H., Matsudaira, P., Mitchison, T. J., and Weitz, D. A. (2004). Colloid surface chemistry critically affects multiple particle tracking measurements of biomaterials. Biophysical Journal, 86(6):4004-4014.

Wang, C. Y. (2003). Stokes slip flow through square and triangular arrays of circular cylinders. Fluid Dynamics Research, 32(5):233-246.

Wang, H., Wu, T. X., Gauza, S., Wu, J. R., and Wu, S.-T. (2006). A method to estimate the leslie coefficients of liquid crystals based on mbba data. Liquid crystals, 33(1):91-98.

Wang, N., Butler, J. P., and Ingber, D. E. (1993). Mechanotransduction across the cell surface and through the cytoskeleton. Science, 260(5111):1124-1127.

Yanai, M., Butler, J. P., Suzuki, T., Sasaki, H., and Higuchi, H. (2004). Regional rheological differences in locomoting neutrophils. American Journal of Physiology - Cell Physiology, 287(3):C603-C611. 